\title{
A reformulated hardening soil model
}

Thomas A. Bower MEng, AFHEA, PhD

Software Engineer, LUSAS, Forge House, Kingston Upon Thames, UK (corresponding author: bowerta@cardiff.ac.uk) (Orcid:0000-0002-0982-0895)

Anthony D. Jefferson MIStructE, CEng, BSC, MSC, PhD

Professor, School of Engineering, Cardiff University, Queen's Buildings, The Parade, Cardiff, UK (Orcid:0000-0002-2050-2521)

\author{
Peter J. Cleall BEng, PhD \\ Reader, School of Engineering, Cardiff University, Queen's Buildings, \\ The Parade, Cardiff, UK (Orcid:0000-0002-4005-5319)
}

The hardening soil (HS) model is an advanced soil plasticity model, which incorporates many features including stiffness stress dependency, hardening from initial loading and soil dilatancy. In this paper, the HS model is explored in depth and two improvements are proposed. The first is a new shear yield surface and hardening rule that have been reformulated to remove singularities. The second is a robust implicit return mapping scheme. Options for improving the global convergence of finite-element analyses are also explored. Single-elements tests replicate results from experimental triaxial data and previous versions of the HS model very closely, and at excellent convergence rates. In addition, a slope stability analysis is performed using the $\varphi-c$ strength reduction method in two-dimensional plane strain. The results from the slope analysis showed good agreement with analytical and graphical slope stability methods. A three-dimensional (3D) slope stability analysis was also conducted with modified boundary conditions, in order to demonstrate the 3D capabilities of the model.

$\begin{array}{ll}\text { Notation } \\ c & \text { cohesion } \\ \boldsymbol{D}_{\mathrm{e}} & \text { elastic stiffness matrix } \\ \boldsymbol{D}_{\mathrm{ep}} & \text { pseudo-consistent tangent matrix } \\ E_{\mathrm{i}}^{\text {ref }} & \text { reference initial modulus } \\ E_{\mathrm{ur}}^{\text {ref }} & \text { reference unload-reload modulus } \\ m & \text { stiffness stress-dependency exponent } \\ p & \text { mean stress } \\ p_{\mathrm{p}} & \text { pre-consolidation pressure } \\ q_{\mathrm{a}} & \text { asymptotic shear stress } \\ q_{\mathrm{f}} & \text { failure shear stress } \\ \alpha & \text { cap shape parameter } \\ \gamma & \text { shear strain } \\ \gamma_{\mathrm{p}} & \text { plastic shear strain } \\ \Delta E & \text { change in Young's modulus between iterations } \\ \Delta \varepsilon & \text { strain increment vector } \\ \Delta \lambda & \text { plasticity multiplier } \\ \delta \lambda & \text { variation in plasticity multiplier } \\ \varepsilon & \text { strain vector } \\ \varepsilon^{\mathrm{p}} & \text { plastic strain vector } \\ \varepsilon_{\mathrm{V}} & \text { volumetric strain } \\ \varepsilon_{\mathrm{v}}^{\mathrm{p}} & \text { plastic volumetric strain } \\ \theta & \text { Lode angle } \\ \rho & \text { density } \\ \sigma & \text { stress vector } \\ \sigma_{\mathrm{LC}} & \text { last converged stress } \\ \sigma^{\text {ref }} & \text { reference stress } \\ \sigma_{\mathrm{tr}} & \text { trial stress } \\ \varphi & \text { friction angle } \\ \varphi_{\mathrm{c}} & \text { critical state friction angle } \\ \varphi_{\mathrm{m}} & \text { mobilised friction angle } \\ & \end{array}$

$\begin{array}{ll}\chi & \text { cap-shaping parameter } \\ \psi & \text { dilatancy angle } \\ \psi_{\mathrm{m}} & \text { mobilised dilatancy angle }\end{array}$

\section{Introduction}

The hardening soil (HS) model is a soil plasticity model, which is gaining increasing popularity in research and design and has been implemented into commercial finite-element codes such as Plaxis (Plaxis, 2016), ZSoil (Obrzud, 2010) and FLAC3D (Jiang and Zhang, 2012). This is a multiple surface plasticity model, which combines many advanced features such as stress-dependent stiffness, non-linear dilatancy progression and separate mechanisms for controlling the shear and the volumetric behaviour of the soil.

The parameters used in the model are easily obtainable from standard soil laboratory tests. To date, several versions of the model have been created; the model was first proposed by Schanz et al. (1999). This version makes use of a hexagonal Mohr-Coulomb-type cone surface, which hardens according to a rule based on the hyperbolic soil model (Duncan and Chang, 1970). It also features an elliptical cap surface, similar to that used in the modified cam-clay model (Roscoe and Burland, 1968).

Modifications to this model were proposed by Benz (2007) and Benz et al. (2008), which addressed the non-smooth failure criterion used in the model, citing that the new failure criterion better captures the experimental failure criterion observed in soils (Matsuoka and Nakai, 1974). This improvement is 
explained by the contribution of the intermediate principal stress to failure. Another part of the work by Benz (2007) was the introduction of a small-strain stiffness overlay to the HS model. Although this small-strain stiffness phenomenon is typically observed in dynamic problems, it was shown that it can improve the results of large boundary-value static problems.

Marcher and Vermeer (2001) introduced softening in the HS model by implementing a void ratio-dependent friction angle. Another modification was made by Truty and Obrzud (2015), which studied the predictions of undrained behaviour in the HS model.

This paper describes the development of a new version of the HS model, hereinafter referred to as the HS-LC model (Lusas-Cardiff University). The innovations in the new version of the model include a modified shear yield criterion, which in the HS-LC model is based on the smooth failure criterion by Matsuoka and Nakai (Matsuoka and Nakai, 1974; Panteghini and Lagioia, 2013). The size of the shear cone is controlled by a new hardening rule, which is based on the triaxial shear hyperbola by Duncan and Chang (1970). A robust return mapping procedure is also implemented, which reduces the residuals of stress, plastic strain, and the hardening variables. This model is then verified against previous versions of the HS model, against published experimental data, and a slope stability analysis is performed.

\section{Conventions}

This paper follows the sign convention of general solid mechanics, where tensile stresses and strains are taken as positive. The principal stresses are ordered in a descending manner $\sigma_{1} \geq \sigma_{2} \geq \sigma_{3}$. Vectors and matrices are denoted in bold. The unique terms of the three-dimensional (3D) stress tensor are represented in the vector

1. $\sigma=\left[\begin{array}{llllll}\sigma_{x} & \sigma_{y} & \sigma_{z} & \sigma_{x y} & \sigma_{y z} & \sigma_{x z}\end{array}\right]^{\mathrm{T}}$

and similarly for the strain tensor $\boldsymbol{\varepsilon}$. Several stress invariants are used throughout this paper to describe the HS and HS-LC models. The mean stress is calculated from the normal stress components

2. $p=\frac{\sigma_{x}+\sigma_{y}+\sigma_{z}}{3}$

The shear stress is defined as

3. $q=\sqrt{\frac{\left(\sigma_{x}-\sigma_{y}\right)^{2}+\left(\sigma_{y}-\sigma_{z}\right)^{2}+\left(\sigma_{z}-\sigma_{x}\right)^{2}}{2}+3\left(\sigma_{x y}^{2}+\sigma_{y z}^{2}+\sigma_{x z}^{2}\right)}$
The third deviatoric stress invariant is the determinant of the deviatoric stress tensor

$$
\begin{aligned}
J_{3}= & \left(\sigma_{x}-p\right)\left[\left(\sigma_{y}-p\right)\left(\sigma_{z}-p\right)-\sigma_{y z}^{2}\right] \\
& -\sigma_{x y}\left[\sigma_{x y}\left(\sigma_{z}-p\right)-\sigma_{y z} \sigma_{x z}\right] \\
& +\sigma_{x z}\left[\sigma_{x y} \sigma_{y z}-\left(\sigma_{y}-p\right) \sigma_{x z}\right]
\end{aligned}
$$

The Lode angle is calculated from the shear stress and third deviatoric stress invariant

5. $\theta=\frac{1}{3} \arcsin \left(\frac{-27 J_{3}}{2 q^{3}}\right)$

and is equal to $\pi / 6$ in triaxial compression $\left(\sigma_{1}=\sigma_{2}>\sigma_{3}\right)$. The volumetric strain is the sum of the normal strains

6. $\varepsilon_{\mathrm{V}}=\varepsilon_{x}+\varepsilon_{y}+\varepsilon_{z}$

The shear strain, as defined by Wood (1990) is given as

7. $\gamma=\sqrt{\frac{2}{9}\left[\left(\varepsilon_{x}-\varepsilon_{y}\right)^{2}+\left(\varepsilon_{y}-\varepsilon_{z}\right)^{2}+\left(\varepsilon_{z}-\varepsilon_{x}\right)^{2}\right]+\frac{1}{3}\left[\varepsilon_{x y}^{2}+\varepsilon_{y z}^{2}+\varepsilon_{x z}^{2}\right]}$

\section{Background to the HS model}

The HS model is a non-linear soil plasticity model used for the analysis of granular and cohesive soils, which utilises three main yield surfaces

- A shear surface which hardens from initial loading

- A cap surface to control volumetric strains

- A Mohr-Coulomb failure envelope to impose a final limit on shear stress.

Benz (2007) suggested the replacement of the hexagonal Mohr-Coulomb-type yield surface with the smooth variation by Matsuoka and Nakai (1974), which produces the same results as the Mohr-Coulomb surface in triaxial conditions when the Lode angle $\theta= \pm \pi / 6$. The main advantages of this smoothed surface are the consideration of the intermediate principal stress at soil failure, and are more computationally convenient due to a reduction in the number of edges and corners, which define the yield surface.

The surfaces in each version were formulated to follow the triaxial hyperbola (Equation 8) proposed by Duncan and Chang (1970) (Figure 1). 


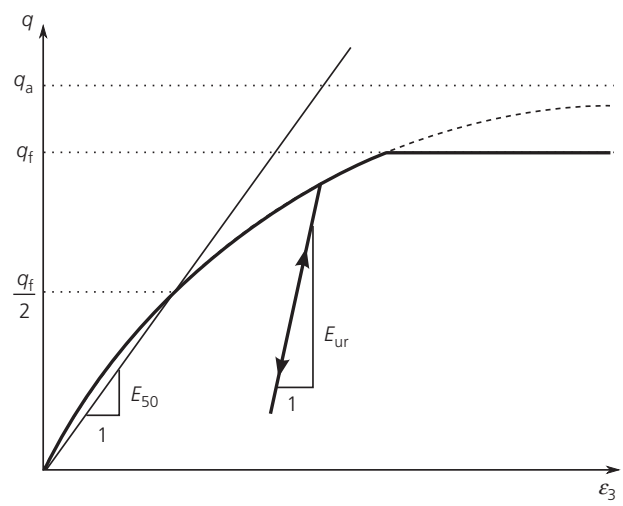

Figure 1. Triaxial compression shearing hyperbola as used in the formulation of HS model (source: redrawn from Benz, 2007)

The equation of the hyperbola in Figure 1 is given by

8. $\varepsilon_{3}=\frac{q_{\mathrm{a}}}{2 E_{50}} \frac{q}{q-q_{\mathrm{a}}}$

The shear stress $q$ is limited to the failure shear stress $q_{\mathrm{f}}$ (given by the Mohr-Coulomb criterion)

9. $q_{\mathrm{f}}=\frac{2 \sin \varphi}{1-\sin \varphi}\left(-\sigma_{1}+c \cot \varphi\right)$

where $c$ is the effective cohesion and $\varphi$ is the friction angle at failure. $E_{50}$ is the secant stiffness at $50 \%$ mobilised shear stress (see Figure 1). The asymptotic shear stress $q_{\mathrm{a}}$ is given by

10. $q_{\mathrm{a}}=\frac{q_{\mathrm{f}}}{R_{\mathrm{f}}}$

where $R_{\mathrm{f}}$ is the failure ratio, which describes the ratio of the failure shear stress to the asymptotic shear stress. A set of stress-dependent stiffness parameters is used throughout the model. These are based on a reference stress $\sigma^{\text {ref }}$ and a stiffness stress-dependency exponent $m$.

11. $E_{\mathrm{ur}}=E_{\mathrm{ur}}^{\mathrm{ref}}\left(\frac{-\sigma_{1}+c \cot \varphi}{-\sigma^{\mathrm{ref}}+c \cot \varphi}\right)^{m}$

12. $E_{50}=E_{50}^{\mathrm{ref}}\left(\frac{-\sigma_{1}+c \cot \varphi}{-\sigma^{\mathrm{ref}}+c \cot \varphi}\right)^{m}$

$E_{\mathrm{ur}}^{\mathrm{ref}}$ and $E_{50}^{\mathrm{ref}}$ are the reference moduli for unloading/reloading and $50 \%$ failure shear stress, respectively, these are the given values at $\sigma^{\text {ref }}$. It is worth noting that a constant stiffness can be achieved by setting $m=0$.
The dilatancy angle $\psi_{\mathrm{m}}$ is also dependent on stress in the HS model; this is used in the plastic potential function to define the gradients of stress return to the shear yield surface. Schanz et al. (1999) used Rowe's stress dilatancy theory (Equation 13) (Rowe, 1962), and Benz (2007) suggested other alternatives (Li and Dafalias, 2000; Søreide, 1990; Wehnert, 2006).

13. $\quad \sin \psi_{\mathrm{m}}=\max \left(0, \frac{\sin \varphi_{\mathrm{m}}-\sin \varphi_{\mathrm{c}}}{1-\sin \varphi_{\mathrm{m}} \sin \varphi_{\mathrm{c}}}\right)$

The critical state friction angle $\varphi_{\mathrm{c}}$ is given by

14. $\quad \sin \varphi_{\mathrm{c}}=\frac{\sin \varphi-\sin \psi}{1-\sin \varphi \sin \psi}$

Additionally, the mobilised friction angle $\varphi_{\mathrm{m}}$ when using the Mohr-Coulomb model is

15. $\quad \sin \varphi_{\mathrm{m}}=\frac{\sigma_{1}-\sigma_{3}}{2 c \cot \varphi-\sigma_{1}-\sigma_{3}}$

Benz (2007) used an alternative definition of the mobilised friction angle to match the Matsuoka-Nakai criterion (Matsuoka and Nakai, 1974).

The shear surface in the HS model is formulated by first taking a position on the hyperbola defined in Equation 8, this is the first two fractions of Equation 16. A full unloading step to $q=0$ is then subtracted, this is the third fraction. The remainder of this unloading is defined by the accumulated plastic shear strain $\gamma_{\mathrm{p}}$, and is used as a hardening parameter for this surface.

16. $f_{\mathrm{s}}=\frac{q_{\mathrm{a}}}{2 E_{50}} \frac{q}{q_{\mathrm{a}}-q}-\frac{q}{E_{\mathrm{ur}}}-\gamma_{\mathrm{p}}=0$

Note that the definition in Equation 16 is different to those quoted by Schanz et al. (1999) and Benz (2007) due to the different definition of shear strain (Equation 7). This hardening cone is active until the shear stress $q$ reaches the failure shear stress $q_{\mathrm{f}}$. It is important to note that the shear yield surface (Equation 16) continues to harden after the failure shear stress is exceeded whenever the minor principal stress $\sigma_{1}$ increases. Therefore, with the HS model, it is necessary to check for yielding of the hardening shear surface, even if the failure criterion has been exceeded previously.

In the two prior version of the HS model, the failure surface is defined by either the Mohr-Coulomb criterion (Equation 17) 
or the Matsuoka-Nakai criterion (Equation 18)

17. $f_{\mathrm{MC}}=\frac{\sigma_{1}+\sigma_{3}}{2} \sin \varphi+\frac{\sigma_{1}-\sigma_{3}}{2}+c \cos \varphi=0$

18. $f_{\mathrm{MN}}=\frac{I_{1} I_{2}}{I_{3}}-\left(9+8 \tan ^{2} \varphi\right)=0$

where $I_{1}, I_{2}$ and $I_{3}$ are the first, second and third invariants of the stress tensor. The first advantage of using the MatsuokaNakai criterion is that the intermediate principal stress is considered; second, the smooth yield is more convenient computationally, as fewer corners/edges are present than in the Mohr-Coulomb yield surface.

The plastic potential used with both of the shear yield surfaces is the circular cone presented by Drucker et al. (1952)

19. $g_{\mathrm{s}}=q+(p-c \cot \varphi) \frac{6 \sin \psi_{\mathrm{m}}}{3-\sin \psi_{\mathrm{m}}}$

The internal angle of the cone is determined by the mobilised dilatancy angle $\psi_{\mathrm{m}}$. Alternate shear plastic potential functions may also be implemented with the HS model, as use of different potential functions has been shown to change the model behaviour; in particular the treatment of the Lode angle after failure (Lagioia and Panteghini, 2014).

The cap surface takes the form of an ellipse in $p-q$ space

20. $f_{\mathrm{c}}=p^{2}+\left(\frac{q}{\chi \alpha}\right)^{2}-p_{\mathrm{p}}^{2}=0$

The steepness of the cap is controlled using the parameter $\alpha$, and $\chi$ is a Lode angle-dependent variable (defined later in Equation 35), which varies the shape of the cap surface in the deviatoric plane such that the locus of its intersection with the shear surface lies on the plane perpendicular to the isotropic compression line $\sigma_{1}=\sigma_{2}=\sigma_{3}$. The pre-consolidation pressure $p_{\mathrm{p}}$ is used as a hardening parameter and controls the overall size of the cap. The cap surface uses an associated flow rule.

\section{Changes to the HS model}

There are a number of aspects of the existing HS formulations that can give rise to numerical difficulties. The first issue relates to the form of the shear yield surface (Equation 16). In many return mapping schemes it is necessary to calculate a 'trial stress', which is obtained by using elastic stiffness for the given strain increment. If the trial shear stress exceeds the asymptotic shear stress - that is, $q>q_{\mathrm{a}}$, then the yield function would become negative, and the return mapping algorithms could not detect the yielding on the shear surface, as shown in Figure 2. Additionally, a trial stress of $q=q_{\mathrm{a}}$ creates a division by zero in the shear yield surface. This issue has also been noted by Cocco and Ruiz (2018). To overcome this difficulty, the shear surface has been reformulated so that it remains positive for any shear stress higher than yield, thus allowing implementation of the HS model in stress return mapping schemes such as closest point projection (CPP).

The use of a second surface to define failure has also been removed in this reformulation, as the mating of a hardening yield surface with a fixed failure surface was found to create numerical difficulties. Instead, soil failure is incorporated into the hardening function of the reformulated shear yield surface.

The next issue is the incremental form of the shear hardening parameter $\gamma_{p}$ as it was found to cause the global NewtonRaphson scheme to converge at an increasingly slower rate throughout load incrementation. It was discovered that this was due to drifting of the solution; the hardening parameter represents the plastic shear strain, and with continued shear strain steps, the hardening parameter would tend to deviate from the plastic strains calculated in the return mapping procedure. This issue is addressed by using the plastic strains directly in the yield function.

Benz (2007) documents use of the CPP method for one and two surface stress returns. However, in this formulation, only the residual for the stresses are reduced during each iteration and the return mapping procedure is based on incremental relationships. In the algorithm presented in this paper, residuals for the plastic strain and the hardening variables are

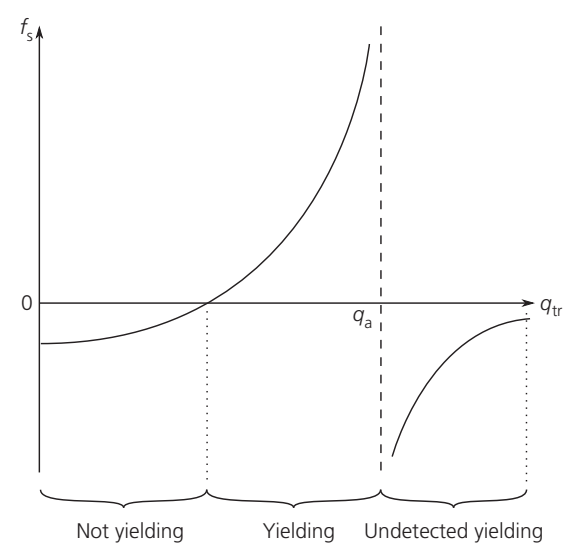

Figure 2. Shear yield function value against shear stress. Yielding is not detected when the trial shear stress $q_{\mathrm{tr}}$ exceeds $q_{\mathrm{a}}$ 
also reduced, leading to algorithm which converges faster. Additionally, the incremental cap-hardening residual is taken into account in the new algorithm. The use of a relationship between total rather than incremental terms reduces the tendency for the solution to drift from the governing equations and reduces step size-dependency.

\subsection{Shear surface}

The following section details the steps taken to reformulate the shear surface in the HS-LC model. In triaxial stress states, the relationship between the axial plastic strain and shear stress is given by Kondner's hyperbola (Equation 8)

21. $\quad \varepsilon_{3}^{\mathrm{p}}=\frac{q_{\mathrm{a}}}{2 E_{50}} \frac{q}{q-q_{\mathrm{a}}}+\frac{q}{E_{\mathrm{ur}}}$

In triaxial stress states $(\theta= \pm \pi / 6)$, the minor and intermediate principal strains are equal $\left(\varepsilon_{1}=\varepsilon_{2}\right)$, substituting this relationship into the definition of the shear strain (Equation 7) gives the triaxial plastic shear strain.

22. $\gamma^{\mathrm{p}}=\frac{2}{3}\left(\varepsilon_{1}-\varepsilon_{3}\right)$

As the hardening of the shear yield function is related solely to plastic shear strain, a state of pure shear is considered - that is

23. $\varepsilon_{\mathrm{v}}^{\mathrm{p}}=\varepsilon_{1}^{\mathrm{p}}+\varepsilon_{1}^{\mathrm{p}}+\varepsilon_{3}^{\mathrm{p}}=0$

which leads to the relationship

24. $\varepsilon_{1}^{\mathrm{p}}=-\frac{1}{2} \varepsilon_{3}^{\mathrm{p}}$

Substituting Equation 24 into Equation 22 gives the relation between plastic triaxial shear strain and axial strain.

25. $\gamma^{\mathrm{p}}=-\varepsilon_{3}^{\mathrm{p}}$

Substituting Equation 25 back into Equation 21 gives the relation between the plastic shear strain and hyperbola used in the HS-LC model.

26. $0=\frac{q_{\mathrm{a}}}{2 E_{50}} \frac{q}{q_{\mathrm{a}}-q}-\frac{q}{E_{\mathrm{ur}}}-\gamma^{\mathrm{p}}$

To obtain a normalised hardening function which has the limits 0 and 1 , the function is formulated to equal the ratio of the shear stress and the asymptotic shear stress. Letting $r_{\mathrm{q}}=q / q_{\mathrm{a}}$ and $r_{\mathrm{u}}=E_{\mathrm{ur}} / 2 E_{50}$ and through manipulation of Equation 26 the following hardening function is obtained

27. $r_{\mathrm{q}}=\frac{1}{2} \sqrt{\left(r_{\mathrm{u}}-1+\frac{\gamma^{\mathrm{p}} E_{\mathrm{ur}}}{q_{\mathrm{a}}}\right)^{2}-\frac{4 \gamma^{\mathrm{p}} E_{\mathrm{ur}}}{q_{\mathrm{a}}}}-\frac{1}{2}\left(r_{\mathrm{u}}-1+\frac{\gamma^{\mathrm{p}} E_{\mathrm{ur}}}{q_{\mathrm{a}}}\right)$

To enforce the shear failure limit at a point below the asymptotic shear stress $q_{\mathrm{a}}$, the parameter $R_{\mathrm{f}}$ is used as a maximum value - that is, $r_{\mathrm{q}} \leq R_{\mathrm{f}}$. The hardening function with the imposed upper limit is plotted in Figure 3 for different values of $q_{\mathrm{a}}$. Intuitively, a higher asymptotic shear stress requires a higher plastic shear strain to reach the failure limit. To maintain stable convergence when $q>q_{\mathrm{f}}$ for standard NewtonRaphson solvers, a very small gradient can be given to the post-failure curve in Figure 3. This gradient is generally only necessary for load-controlled analyses.

The 'loading' and 'failure' surfaces are combined with the latter being the limit of the former. The chosen shear yield function employs Panteghini and Lagioia's reformulated version of the Matsuoka-Nakai failure surface (Panteghini and Lagioia, 2013). The yield function is given by

28. $f_{\mathrm{s}}=q+\frac{M r_{\mathrm{q}}}{\rho R_{\mathrm{f}}}(p-c \cot \varphi)=0$

in which $\rho$ is the Lode angle-dependent parameter which alters the shape of the yield surface in the $\pi$-plane (Equation 32). $M$ controls the apex angle of the cone (Equation 33); when $r_{\mathrm{q}}=R_{\mathrm{f}}$, Equation 28 is equivalent to the Matsuoka-Nakai failure surface (Equation 18). The definitions of each required intermediate parameter are listed below

29. $\eta=\frac{2 \sin \varphi}{\sqrt{3+\sin ^{2} \varphi}}$

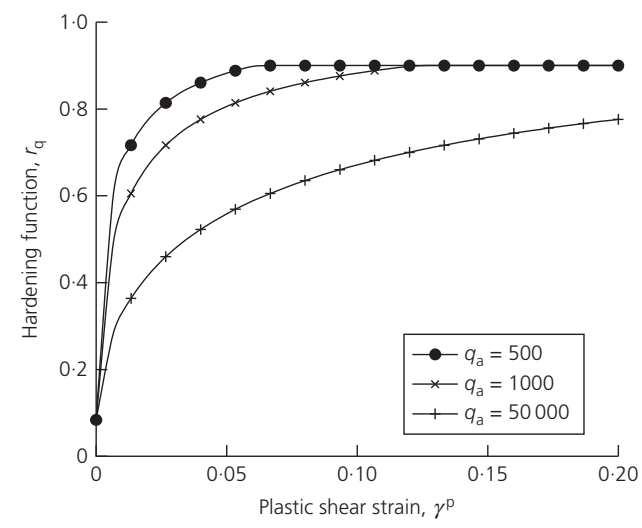

Figure 3. Shear surface hardening function with plastic shear strain with $R_{f}=0.9$ 
30. $\xi=\frac{\sin \varphi\left(9-\sin ^{2} \varphi\right)}{\left(3+\sin ^{2} \varphi\right)^{1 \cdot 5}}$

The Lode angle dependency is given by

31. $\Theta(\theta)=2 \sqrt{3} \cos \left[\frac{1}{3} \arccos (\xi \sin (-3 \theta))\right]$

The parameter $\rho$ normalises the Lode angle dependency to triaxial compression. The last converged stress is used in this calculation.

32. $\rho=\frac{\Theta\left(\theta_{\mathrm{LC}}\right)}{\Theta(\pi / 6)}$

The parameter $M$ is given by

33. $M=\frac{3 \sqrt{3} \eta}{\Theta(\pi / 6)}$

This surface uses a non-associated flow rule. The plastic potential is that of Drucker et al. (1952), as described in Equation 19. The mobilised friction angle is also redefined to match the current failure criterion. This is used in the calculation of the mobilised dilatancy angle $\sin \psi_{\mathrm{m}}$ (Equation 13)

34. $\quad \sin \varphi_{\mathrm{m}}=\frac{3 q}{6 \chi(-p+c \cot \varphi)+q}$

where $\chi$ is equivalent to the Lode dependency term described by Schanz et al. (1999) and Benz (2007), and it relates to the terms in this proposed model by the following relationship

35. $\chi=\frac{M}{\rho}\left(\frac{3-\sin \varphi}{6 \sin \varphi}\right)$

The shaping parameter $\chi$ is used in the cap yield surface (Equation 20).

\subsection{Tension surface}

A tension limit $\sigma_{\mathrm{t}}$ is imposed on the mean stress in this proposed model as follows

36. $f_{\mathrm{t}}=p-\sigma_{\mathrm{t}}=0$

where $\sigma_{\mathrm{t}}$ is the tension cutoff and is entered as a material parameter. The tension yield function uses an associated flow rule $\left(g_{\mathrm{t}} \equiv f_{\mathrm{t}}\right)$ and does not harden.
One of the previous versions of the HS model that Benz (2007) made use of was a Rankine-type criterion to handle tensile stresses. In this formulation, the mean stress criterion is used for computational convenience to minimise the number of edges and corners between the yield surfaces.

\section{The CPP method}

The CPP method (a type of backward Euler method) is an implicit return mapping scheme (Simo and Hughes, 2006) whereby the returned stress path is based on the gradients at the final converged stress state (Figure 4).

A trial stress $\sigma_{\mathrm{tr}}$ is calculated using the elastic stiffness matrix $\boldsymbol{D}_{\mathrm{e}}$, the stress at the last converged state $\boldsymbol{\sigma}_{\mathrm{LC}}$ and the total strain increment $\Delta \varepsilon$

37. $\sigma_{\mathrm{tr}}=\sigma_{\mathrm{LC}}+D_{\mathrm{e}} \Delta \varepsilon$

The aim of the CPP method is to satisfy the following conditions

38. $f(\boldsymbol{\sigma}, \boldsymbol{\mu}) \leq 0$

39. $\boldsymbol{R}=0$

40. $\Delta \lambda \geq 0$

41. $\Delta \lambda f(\boldsymbol{\sigma}, \boldsymbol{\mu})=0$

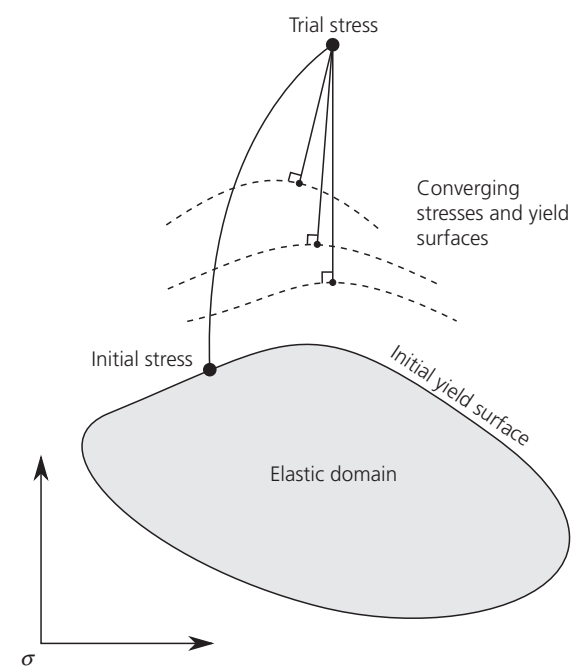

Figure 4. CPP method in 2D stress space (associated flow rule) 
where $f(\boldsymbol{\sigma}, \boldsymbol{\mu})=0$ is the yield function, which forms a surface in $3 \mathrm{D}$ stress space, $\boldsymbol{\sigma}$ is the converged stress state, $\boldsymbol{\mu}$ represents one or more state variables, which define the position of the yield surface, $\boldsymbol{R}$ is the residual of the plastic strains and $\Delta \lambda$ is the variation in the plasticity multiplier which governs the size of the plastic strain steps.

As there are several yield surfaces present in the HS model, and the evolution rules take different forms, a separate CPP algorithm is required for each yield surface and each possible combination of yield surfaces. The formulation of the CPP algorithms is documented in the following sections.

\subsection{Single surface stress return}

The role of the CPP algorithm for a single surface is to satisfy one yield criterion.

42. $f\left(\boldsymbol{\sigma}, \boldsymbol{\sigma}_{\mathrm{LC}}, \varepsilon^{\mathrm{p}}\right)=0$

The plastic strain residual is given by

43. $\boldsymbol{R}=-\Delta \varepsilon^{\mathrm{p}}+\Delta \lambda \frac{\partial g}{\partial \boldsymbol{\sigma}}=0$

Expanding Equation 43 as a Taylor's series gives

44. $\quad \boldsymbol{R}+\boldsymbol{\delta} \boldsymbol{R}=\boldsymbol{R}-\boldsymbol{\delta} \varepsilon^{\mathrm{p}}+\delta \lambda \frac{\partial g}{\partial \boldsymbol{\sigma}}+\Delta \lambda \frac{\partial^{2} g}{\partial \boldsymbol{\sigma}^{2}} \delta \boldsymbol{\sigma}=0$

Rearranging to obtain the plastic strain increment

45. $\quad \delta \varepsilon^{\mathrm{p}}=\boldsymbol{R}+\delta \lambda \frac{\partial g}{\partial \boldsymbol{\sigma}}+\Delta \lambda \frac{\partial^{2} g}{\partial \boldsymbol{\sigma}^{2}} \boldsymbol{\delta} \boldsymbol{\sigma}$

Expanding Equation 42 as a Taylor's series

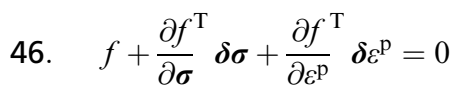

Substituting the plastic strain increment (Equation 45) into Equation 46

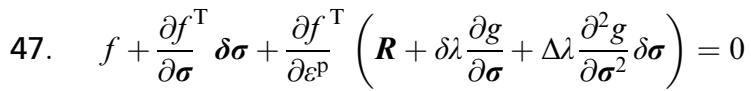

Grouping the $\delta \boldsymbol{\sigma}$ terms gives

48. $f+\boldsymbol{F}^{\mathrm{T}} \boldsymbol{\delta} \boldsymbol{\sigma}+\frac{\partial s^{\mathrm{T}}}{\partial \varepsilon^{\mathrm{p}}}\left(\boldsymbol{R}+\delta \lambda \frac{\partial g}{\partial \boldsymbol{\sigma}}\right)=0$ where

49. $\quad \boldsymbol{F}^{\mathrm{T}}=\frac{\partial f^{\mathrm{T}}}{\partial \boldsymbol{\sigma}}+\Delta \lambda \frac{\partial f^{\mathrm{T}}}{\partial \varepsilon^{\mathrm{p}}} \frac{\partial^{2} g}{\partial \boldsymbol{\sigma}^{2}}$

During the CPP iterations, the change in stress between iterations is

50. $\delta \boldsymbol{\sigma}=-\boldsymbol{D}_{\mathrm{e}} \boldsymbol{\delta} \varepsilon^{\mathrm{p}}$

Substituting the plastic strain increment (Equation 45) into Equation 50

51. $\delta \boldsymbol{\sigma}=-\boldsymbol{D}_{\mathrm{e}}\left(\boldsymbol{R}+\delta \lambda \frac{\partial g}{\partial \boldsymbol{\sigma}}+\Delta \lambda \frac{\partial^{2} g}{\partial \boldsymbol{\sigma}^{2}} \delta \boldsymbol{\sigma}\right)$

Rearranging Equation 51 gives

52. $\delta \boldsymbol{\sigma}=-\boldsymbol{A}_{\mathrm{e}}\left(\boldsymbol{R}+\delta \lambda \frac{\partial g}{\partial \boldsymbol{\sigma}}\right)$

where

53. $\boldsymbol{A}_{\mathrm{e}}=\left(\boldsymbol{I}+\Delta \lambda \boldsymbol{D}_{\mathrm{e}} \frac{\partial^{2} g}{\partial \boldsymbol{\sigma}^{2}}\right)^{-1} \boldsymbol{D}_{\mathrm{e}}$

and $\boldsymbol{I}$ is the identity matrix. Finally, the variation in the plasticity multiplier is calculated through substitution of Equation 52 into Equation 48 and rearranging for $\delta \lambda$.

54. $\delta \lambda=\frac{f-\boldsymbol{F}^{\mathrm{T}} \boldsymbol{A}_{\mathrm{e}} \boldsymbol{R}+\left(\partial f / \partial \varepsilon^{\mathrm{p}}\right)^{\mathrm{T}} \boldsymbol{R}}{\boldsymbol{F}^{\mathrm{T}} \boldsymbol{A}_{\mathrm{e}}(\partial g / \partial \sigma)-\left(\partial f / \partial \varepsilon^{\mathrm{p}}\right)^{\mathrm{T}}(\partial g / \partial \sigma)}$

\subsection{Two surface stress return}

When both surfaces are active, the stress state must return to the line which is the intersection of both surfaces. The formulation must also take into account the inter-dependency between the hardening rules of each surface.

The total plastic strain residual now incorporates hardening from both surfaces

55. $\boldsymbol{R}=-\Delta \varepsilon^{\mathrm{p}}+\Delta \lambda_{1} \frac{\partial g_{1}}{\partial \boldsymbol{\sigma}}+\Delta \lambda_{2} \frac{\partial g_{2}}{\partial \boldsymbol{\sigma}}=0$

Expanding Equation 55 as a Taylor's series and simplifying leads to a form of the plastic strain increment which involves 
both surfaces.

56. $\quad \delta \varepsilon^{\mathrm{p}}=\boldsymbol{R}+\delta \lambda_{1} \frac{\partial g_{1}}{\partial \boldsymbol{\sigma}}+\delta \lambda_{2} \frac{\partial g_{2}}{\partial \boldsymbol{\sigma}}+\left(\Delta \lambda_{1} \frac{\partial^{2} g_{1}}{\partial \boldsymbol{\sigma}^{2}}+\Delta \lambda_{2} \frac{\partial^{2} g_{2}}{\partial \boldsymbol{\sigma}^{2}}\right) \boldsymbol{\delta} \boldsymbol{\sigma}$

The expansion of the yield surfaces remain identical to Equation 46, however, the new definition of the plastic strain increment Equation 56 must be substituted into Equation 46. Grouping the $\boldsymbol{\delta} \boldsymbol{\sigma}$ after this substitution leads to

57. $f_{1}+\boldsymbol{F}_{1}^{\mathrm{T}} \boldsymbol{\delta} \boldsymbol{\sigma}+\frac{\partial f_{1}^{\mathrm{T}}}{\partial \varepsilon^{\mathrm{p}}}\left(\boldsymbol{R}+\delta \lambda_{1} \frac{\partial g_{1}}{\partial \boldsymbol{\sigma}}+\delta \lambda_{2} \frac{\partial g_{2}}{\partial \boldsymbol{\sigma}}\right)=0$

where

58. $\quad \boldsymbol{F}_{1}^{\mathrm{T}}=\frac{\partial f_{1}^{\mathrm{T}}}{\partial \boldsymbol{\sigma}}+\frac{\partial f_{1}^{\mathrm{T}}}{\partial \varepsilon^{\mathrm{p}}}\left(\Delta \lambda_{1} \frac{\partial^{2} g_{1}}{\partial \boldsymbol{\sigma}^{2}}+\Delta \lambda_{2} \frac{\partial^{2} g_{2}}{\partial \boldsymbol{\sigma}^{2}}\right)$

and similarly for the second surface

59. $\quad \boldsymbol{F}_{2}^{\mathrm{T}}=\frac{\partial f_{2}^{\mathrm{T}}}{\partial \boldsymbol{\sigma}}+\frac{\partial f_{2}^{\mathrm{T}}}{\partial \varepsilon^{\mathrm{p}}}\left(\Delta \lambda_{1} \frac{\partial^{2} g_{1}}{\partial \boldsymbol{\sigma}^{2}}+\Delta \lambda_{2} \frac{\partial^{2} g_{2}}{\partial \boldsymbol{\sigma}^{2}}\right)$

The two surface form of the plastic strain increment (Equation 56) is now substituted into the incremental stress relationship (Equation 50)

60. $\delta \boldsymbol{\sigma}=-\boldsymbol{D}_{\mathrm{e}}\left[\boldsymbol{R}+\delta \lambda_{1} \frac{\partial g_{1}}{\partial \boldsymbol{\sigma}}+\delta \lambda_{2} \frac{\partial g_{2}}{\partial \boldsymbol{\sigma}}+\left(\Delta \lambda_{1} \frac{\partial^{2} g_{1}}{\partial \boldsymbol{\sigma}^{2}}+\Delta \lambda_{2} \frac{\partial^{2} g_{2}}{\partial \boldsymbol{\sigma}^{2}}\right) \boldsymbol{\delta} \boldsymbol{\sigma}\right]$

The stress increment from Equation 60 simplifies to

61. $\delta \boldsymbol{\sigma}=-\boldsymbol{A}_{\mathrm{e}}\left(\boldsymbol{R}+\delta \lambda_{1} \frac{\partial g_{1}}{\partial \boldsymbol{\sigma}}+\delta \lambda_{2} \frac{\partial g_{2}}{\partial \boldsymbol{\sigma}}\right)$

where

62. $\boldsymbol{A}_{\mathrm{e}}=\left(\boldsymbol{I}+\Delta \lambda_{1} \boldsymbol{D}_{\mathrm{e}} \frac{\partial^{2} g_{1}}{\partial \boldsymbol{\sigma}^{2}}+\Delta \lambda_{2} \boldsymbol{D}_{\mathrm{e}} \frac{\partial^{2} g_{2}}{\partial \boldsymbol{\sigma}^{2}}\right)^{-1} \boldsymbol{D}_{\mathrm{e}}$

Substituting Equation 61 into Equation 57 and its second surface counterpart, then rearranging into matrix form gives an expression for the plasticity multipliers to be solved.

63. $\boldsymbol{\Omega}_{\mathrm{f}}=\boldsymbol{\Omega}\left[\begin{array}{l}\delta \lambda_{1} \\ \delta \lambda_{2}\end{array}\right]$ where

$$
\boldsymbol{\Omega}_{\mathrm{f}}=\left[\begin{array}{c}
f_{1}-\boldsymbol{F}_{1}^{\mathrm{T}} \boldsymbol{A}_{\mathrm{e}} \boldsymbol{R}+\frac{\partial f_{1}}{\partial \varepsilon^{\mathrm{p}}} \boldsymbol{R} \\
f_{2}-\boldsymbol{F}_{2}^{\mathrm{T}} \boldsymbol{A}_{\mathrm{e}} \boldsymbol{R}+\frac{\partial f_{2}}{\partial \varepsilon^{\mathrm{p}}} \boldsymbol{R}
\end{array}\right]
$$

65. $\boldsymbol{\Omega}=\left[\begin{array}{ll}\boldsymbol{F}_{1}^{\mathrm{T}} \boldsymbol{A}_{\mathrm{e}} \frac{\partial g_{1}}{\partial \sigma}-\frac{\partial f_{1}^{\mathrm{T}}}{\partial \varepsilon^{\mathrm{p}}} \frac{\partial g_{1}}{\partial \sigma} & \boldsymbol{F}_{1}^{\mathrm{T}} \boldsymbol{A}_{\mathrm{e}} \frac{\partial g_{2}}{\partial \sigma}-\frac{\partial f_{1}^{\mathrm{T}}}{\partial \varepsilon^{\mathrm{p}}} \frac{\partial g_{2}}{\partial \sigma} \\ \boldsymbol{F}_{2}^{\mathrm{T}} \boldsymbol{A}_{\mathrm{e}} \frac{\partial g_{1}}{\partial \sigma}-\frac{\partial f_{2}^{\mathrm{T}}}{\partial \varepsilon^{\mathrm{p}}} \frac{\partial g_{1}}{\partial \sigma} & \boldsymbol{F}_{2}^{\mathrm{T}} \boldsymbol{A}_{\mathrm{e}} \frac{\partial g_{2}}{\partial \sigma}-\frac{\partial f_{2}^{\mathrm{T}}}{\partial \varepsilon^{\mathrm{p}}} \frac{\partial g_{2}}{\partial \sigma}\end{array}\right]$

Equation 63 is then solved for the unknown plasticity multipliers $\delta \lambda_{1}$ and $\delta \lambda_{2}$.

\subsection{Additional CPP considerations}

The CPP formulations in this section are described in terms of a generic case where the hardening rule is related directly to the plastic strain vector $\varepsilon^{\mathrm{p}}$. Hence, the algorithms can be used directly for the shear and tension surfaces. The tension surface does not harden, therefore, the hardening term $\left(\partial f_{\mathrm{t}} / \partial \varepsilon^{\mathrm{p}}\right)$ is equal to zero.

The cap surface uses an incremental form for the hardening rule, therefore a hardening residual must be included in the formulation

66. $\quad R_{\mathrm{h}}=-\Delta p_{\mathrm{p}}+\Delta \lambda \frac{\partial p_{\mathrm{p}}}{\partial \varepsilon_{\mathrm{v}}^{\mathrm{p}}} \frac{\partial \varepsilon_{\mathrm{v}}^{\mathrm{p}}}{\partial \varepsilon^{\mathrm{p}}} \frac{\partial g_{\mathrm{c}}}{\partial \boldsymbol{\sigma}}=0$

Taylor's series expansion of the yield function also includes the hardening parameter $p_{\mathrm{p}}$ directly. The plasticity multiplier for the cap surface is calculated using this hardening residual

67. $\delta \lambda_{\mathrm{c}}=\frac{f_{\mathrm{c}}-\boldsymbol{F}_{\mathrm{c}}^{\mathrm{T}} \boldsymbol{A}_{\mathrm{e}} \boldsymbol{R}+\left(\partial f_{\mathrm{c}} / \partial p_{\mathrm{p}}\right) R_{\mathrm{h}}}{\boldsymbol{F}_{\mathrm{c}}^{\mathrm{T}} \boldsymbol{A}_{\mathrm{e}}\left(\partial g_{\mathrm{c}} / \partial \boldsymbol{\sigma}\right)-\left(\partial f_{\mathrm{c}} / \partial p_{\mathrm{p}}\right)\left(\partial p_{\mathrm{p}} / \partial \varepsilon_{\mathrm{v}}^{\mathrm{p}}\right)\left(\partial \varepsilon_{\mathrm{v}}^{\mathrm{p}} / \partial \varepsilon^{\mathrm{p}}\right)^{\mathrm{T}}\left(\partial g_{\mathrm{c}} / \partial \boldsymbol{\sigma}\right)}$

\subsection{Return mapping procedure}

As the HS-LC model uses multiple hardening yield surfaces and uses a non-associated flow rule, a simple zoning method is not sufficient to detect which surfaces are active for a given trial stress. Instead, a more complex approach is adopted which returns the stress to each surface in turn, then checks other surfaces for yielding. Figure 5 illustrates the order in which yield criteria are checked and returned to (following from Benz (2007)). The primary and most dominant surface in the HS and HS-LC models is the shear surface, hence the trial stress state $\sigma_{\mathrm{tr}}$ and plastic strains $\varepsilon^{\mathrm{p}}$ are checked against the shear surface $f_{\mathrm{s}}=0$ (Equation 28). If this yield criterion is 


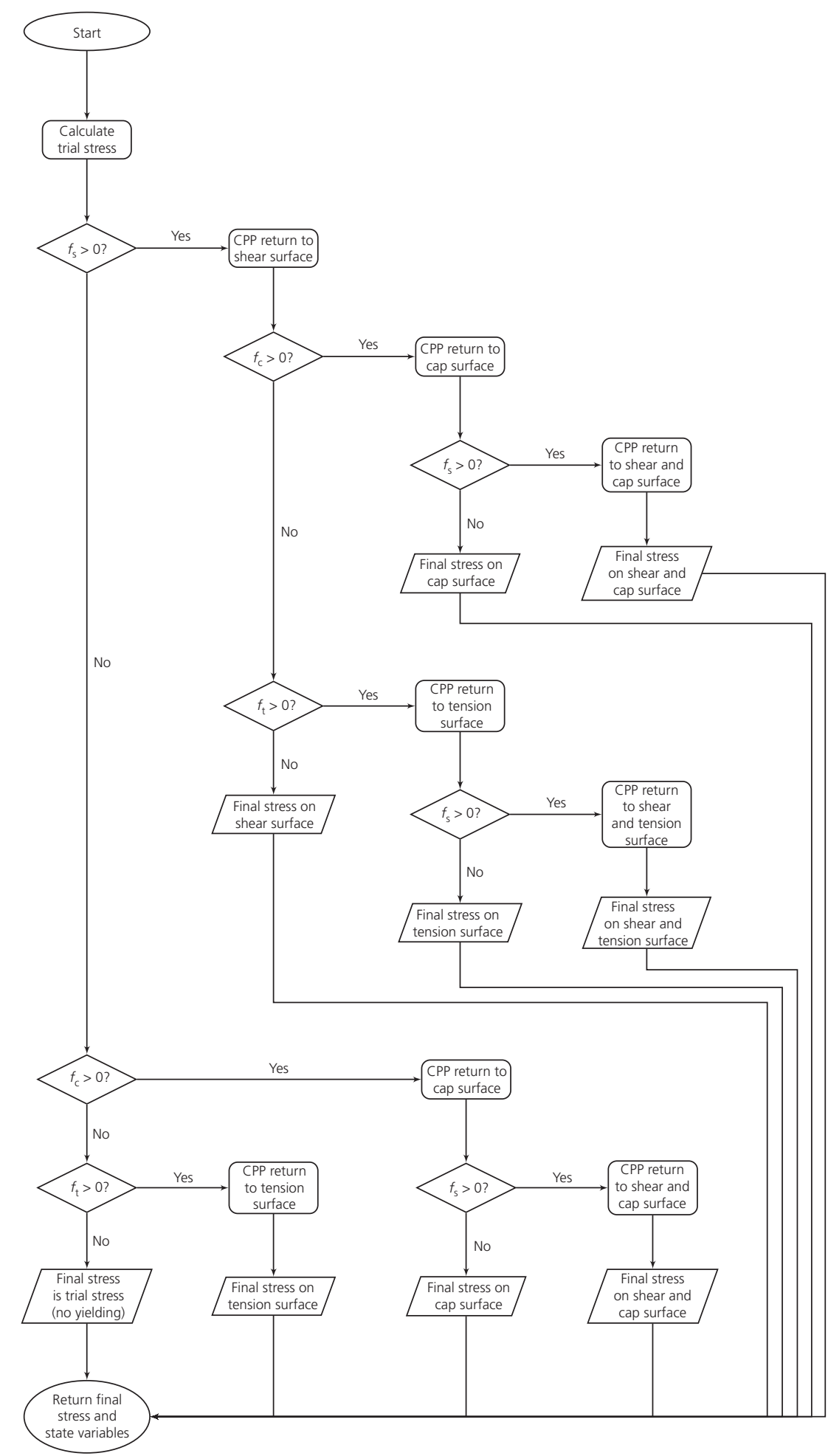

Figure 5. Return mapping algorithm used in the HS-LC model: each yield function and return mapping algorithm are referenced as follows; $f_{\mathrm{s}}$ - Equation 28; $f_{\mathrm{c}}$ - Equation 20; $f_{\mathrm{t}}$ - Equation 36; CPP shear - Equations 42-54 with subscript s; CPP cap - Equations 42-54, subscript C, and hardening residual (Equation 66); CPP tension - Equations 42-54, subscript t; CPP shear and cap - Equations 55-63, subscripts s and C, and cap hardening residual (Equation 66); CPP shear and tension - Equations 55 to 63, subscripts s and t 
exceeded, then the single surface CPP algorithm is used to return the stress state to the shear surface.

After the return to the shear surface, it may be possible that one of the other yield criteria are exceeded. Following a shear surface return, the cap yield function $f_{\mathrm{c}}=0$ (Equation 20) is checked with the returned stress and state variables. If the cap criterion is exceeded, two surface hardening is not automatically assumed, and the stress is returned to the cap surface using the single surface CPP algorithm from the trial stress $\sigma_{\mathrm{tr}}$ and starting state variables. If the shear yield criterion is still exceeded after the cap return, then the two surface CPP algorithm is used. Unless the soil analysed is heavily overconsolidated, this combination of surfaces is the most commonly used in primary loading.

A similar procedure is adopted for the tensile yield surface $f_{\mathrm{t}}=0$ (Equation 36). After the first shear surface return, the tensile criterion is checked. If exceeded, then the single surface CPP algorithm is used to return the stress and state variables to the tension yield surface. If the shear criterion is still exceeded, then the two surface CPP algorithm is used.

In the case that the shear surface is not initially yielding, the cap surface yield criterion is checked and returned to if necessary. This cap return may place the updated stress in a region which exceeds the shear yield criterion. If the shear yield criterion is exceeded, then the two surface CPP algorithm is used to return the stress and state variables to the shear and cap surfaces. The tension surface does not need to be checked here because the tension and cap surfaces cannot both be active.

Finally, if no yielding was detected before this stage, then the tensile yield criterion $f_{\mathrm{t}}=0$ (Equation 36) is checked and returned to if this criterion is exceeded. The shear criterion does not need to be checked again because shear yielding would have been detected at the first stage.

\subsection{Example CPP algorithm}

Algorithm 1 describes the most complex of the CPP algorithms, namely the return to the shear and cap surfaces. It is the most complex because it includes reduction of the cap-hardening residual $R_{\mathrm{h}}$ due to the decoupling of the two surfaces.

To convert this algorithm to return to the shear and tension surfaces, the subscript $c$ is replaced with $t$. The tension surface uses an associated flow rule $\left(g_{\mathrm{t}} \equiv f_{\mathrm{t}}\right)$. Additionally, the tension surface is non-hardening, therefore $\left(\partial f_{\mathrm{t}} / \partial \varepsilon^{\mathrm{p}}\right)=0$ in each of the relevant expressions.

The procedure for the single-surface CPP algorithms is similar to those of the double-surface procedure. The stress increment, plastic strain increment, and pseudo-consistent constitutive matrix contain derivatives for one surface only. The calculation of the plasticity multiplier $\delta \lambda$ is performed using Equation 54.

Algorithm 1 CPP algorithm for HS-LC shear and cap surfaces.

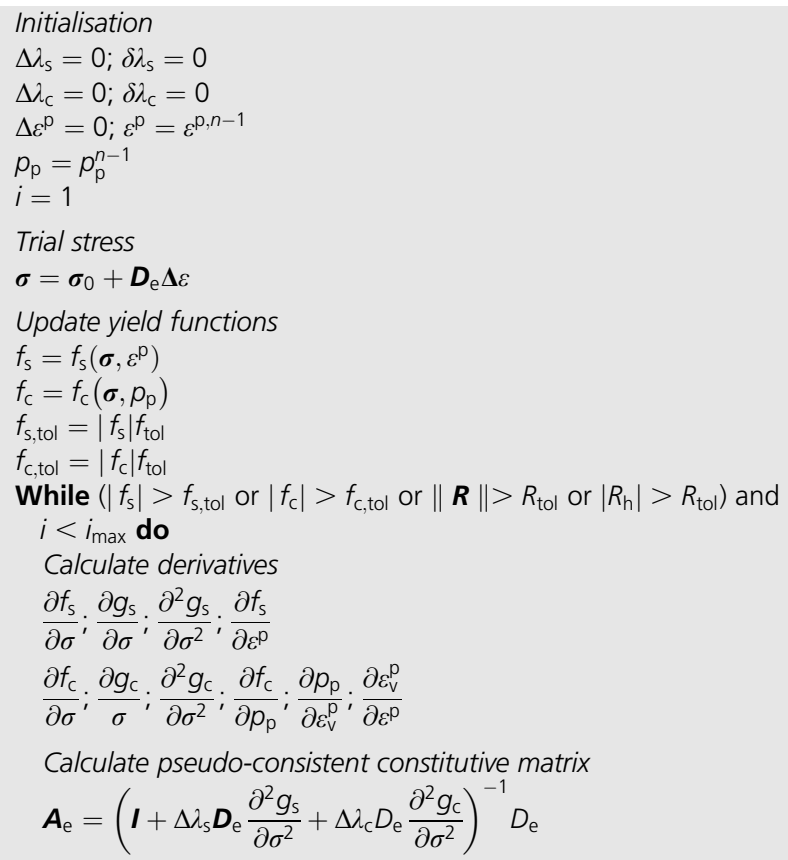

Calculate residual plastic strain and hardening parameter residual $\boldsymbol{R}=-\Delta \varepsilon^{p}+\Delta \lambda_{s} \frac{\partial g_{s}}{\partial \sigma}+\Delta \lambda_{c} \frac{\partial g_{c}}{\partial \sigma}$ $R_{\mathrm{h}}=-\Delta p_{\mathrm{p}}+\Delta \lambda_{\mathrm{c}} \frac{\partial p_{\mathrm{p}}}{\partial \varepsilon_{\mathrm{v}}^{\mathrm{p}}} \frac{\partial \varepsilon_{\mathrm{v}}^{\mathrm{p}}}{\partial \varepsilon^{\mathrm{p}}} \frac{\partial g_{\mathrm{c}}}{\partial \sigma}$

Stress increment $\boldsymbol{\delta} \boldsymbol{\sigma}=-\boldsymbol{A}_{\mathrm{e}}\left(\boldsymbol{R}+\delta \lambda_{\mathrm{s}} \frac{\partial g_{\mathrm{s}}}{\partial \sigma}+\delta \lambda_{\mathrm{c}} \frac{\partial g_{\mathrm{c}}}{\partial \sigma}\right)$

Plasticity multipliers

$\boldsymbol{\Omega}=\left[\begin{array}{cc}\boldsymbol{F}_{\mathrm{S}}^{\top} \boldsymbol{A}_{\mathrm{e}} \frac{\partial g_{\mathrm{S}}}{\partial \sigma}-\frac{\partial f_{\mathrm{S}}^{\top}}{\partial \varepsilon^{\mathrm{p}}} \frac{\partial g_{\mathrm{s}}}{\partial \sigma} & \boldsymbol{F}_{\mathrm{S}}^{\top} \boldsymbol{A}_{\mathrm{e}} \frac{\partial g_{\mathrm{C}}}{\partial \sigma}-\frac{\partial f_{\mathrm{s}}^{\top}}{\partial \varepsilon^{\mathrm{p}}} \frac{\partial g_{\mathrm{C}}}{\partial \sigma} \\ \boldsymbol{F}_{\mathrm{C}}^{\top} \boldsymbol{A}_{\mathrm{e}} \frac{\partial g_{\mathrm{s}}}{\partial \sigma} & \boldsymbol{F}_{\mathrm{C}}^{\top} \boldsymbol{A}_{\mathrm{e}} \frac{\partial g_{\mathrm{C}}}{\partial \sigma}-\frac{\partial f_{\mathrm{C}}}{\partial p_{\mathrm{p}}} \frac{\partial p_{\mathrm{p}}}{\partial \varepsilon_{\mathrm{v}}^{\mathrm{p}}} \frac{\partial \varepsilon_{\mathrm{v}}^{\mathrm{p}}}{\partial \varepsilon^{\mathrm{p}}} \frac{\partial g_{\mathrm{C}}}{\partial \sigma}\end{array}\right]$

$\boldsymbol{\Omega}_{\mathrm{f}}=\left[\begin{array}{c}f_{\mathrm{s}}-\boldsymbol{F}_{\mathrm{s}}^{\top} \boldsymbol{A}_{\mathrm{e}} \boldsymbol{R}+\frac{\partial f_{\mathrm{s}}}{\partial \varepsilon^{\mathrm{p}}} \boldsymbol{R} \\ f_{\mathrm{c}}-\boldsymbol{F}_{\mathrm{c}}^{\top} \boldsymbol{A}_{\mathrm{e}} \boldsymbol{R}+\frac{\partial f_{\mathrm{c}}}{\partial p_{\mathrm{p}}} R_{\mathrm{h}}\end{array}\right]$

$\left[\begin{array}{l}\delta \lambda_{s} \\ \delta \lambda_{c}\end{array}\right]=\Omega^{-1} \Omega_{f}$

$\Delta \lambda_{\mathrm{s}}=\Delta \lambda_{\mathrm{s}}+\delta \lambda_{\mathrm{s}}$

$\Delta \lambda_{c}=\Delta \lambda_{c}+\delta \lambda_{c}$

Update plastic strains

$\boldsymbol{\delta} \varepsilon^{\mathrm{p}}=\boldsymbol{R}+\delta \lambda_{\mathrm{s}} \frac{\partial g_{\mathrm{s}}}{\partial \sigma}+\delta \lambda_{\mathrm{c}} \frac{\partial g_{\mathrm{c}}}{\partial \sigma}+\left(\Delta \lambda_{\mathrm{s}} \frac{\partial^{2} g_{\mathrm{s}}}{\partial \sigma^{2}}+\Delta \lambda_{\mathrm{c}} \frac{\partial^{2} g_{\mathrm{c}}}{\partial \sigma^{2}}\right) \boldsymbol{\delta} \boldsymbol{\sigma}$

$\Delta \varepsilon^{\mathrm{p}}=\boldsymbol{\Delta} \varepsilon^{\mathrm{p}}+\boldsymbol{\delta} \varepsilon^{\mathrm{p}}$ 


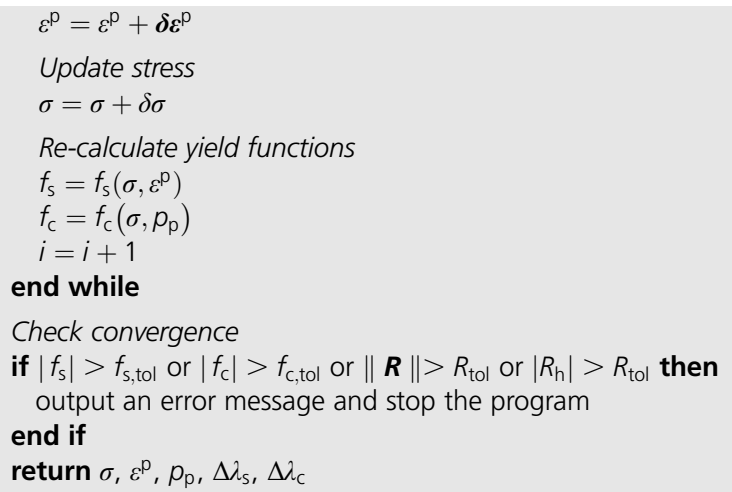

\section{Consistent tangent matrix}

The consistent tangent matrix (CTM) relates infinitesimal stresses and strains. It is used to calculate the stress gradients on one or more active yield surfaces and preserves the quadratic rate of convergence in the global Newton-Raphson iterative scheme.

Much of the theory for defining the consistent tangent matrix has been discussed already, however it is worth noting that in the formulation of the CTM, the stress state is assumed to already be on one or more yield surfaces and the residuals relating to the active surface(s) are zero. The plastic multiplier(s) $\Delta \lambda$ from the previous iteration are also required. The consistent tangent is defined as

68. $\quad D_{\mathrm{ep}}=\frac{\mathrm{d} \sigma}{\mathrm{d} \varepsilon}$

If the stress state lies on a single yield surface, then the consistent tangent is given as

69.

$$
\boldsymbol{D}_{\mathrm{ep}}=\boldsymbol{A}_{\mathrm{e}}-\frac{\boldsymbol{A}_{\mathrm{e}}(\partial g / \partial \boldsymbol{\sigma}) \boldsymbol{F}^{\mathrm{T}} \boldsymbol{A}_{\mathrm{e}}}{\boldsymbol{F}^{\mathrm{T}} \boldsymbol{A}_{\mathrm{e}}(\partial g / \partial \boldsymbol{\sigma})-\left(\partial f / \partial \varepsilon^{\mathrm{p}}\right)^{\mathrm{T}}(\partial g / \partial \boldsymbol{\sigma})}
$$

When two surfaces are active, a more complex approach is used which takes into account the gradients and hardening rules from both surfaces

70

$$
\boldsymbol{D}_{\mathrm{ep}}=\boldsymbol{A}_{\mathrm{e}}-\left(\Omega^{-1}\right)_{1,1} \boldsymbol{A}_{\mathrm{e}} \frac{\partial g_{1}}{\partial \boldsymbol{\sigma}} \boldsymbol{F}_{1}^{\mathrm{T}} \boldsymbol{A}_{\mathrm{e}}-\left(\Omega^{-1}\right)_{1,2} \boldsymbol{A}_{\mathrm{e}} \frac{\partial g_{1}}{\partial \boldsymbol{\sigma}} \boldsymbol{F}_{2}^{\mathrm{T}} \boldsymbol{A}_{\mathrm{e}}
$$

$$
-\left(\Omega^{-1}\right)_{2,1} \boldsymbol{A}_{\mathrm{e}} \frac{\partial g_{2}}{\partial \boldsymbol{\sigma}} \boldsymbol{F}_{1}^{\mathrm{T}} \boldsymbol{A}_{\mathrm{e}}-\left(\Omega^{-1}\right)_{2,2} \boldsymbol{A}_{\mathrm{e}} \frac{\partial g_{2}}{\partial \boldsymbol{\sigma}} \boldsymbol{F}_{2}^{\mathrm{T}} \boldsymbol{A}_{\mathrm{e}}
$$

\section{Additional computational considerations}

During preliminary testing of the HS-LC model, it was found that some step-size dependency occurred when modelling certain simulations. In the initial implementation of the HS -LC model, the Young modulus used in the calculation of the elastic and tangent stiffness matrices was based on the converged stress from the previous increment. Use of the last converged modulus significantly improves the stability of the model, as the non-linearity within a given increment is reduced. For large step sizes, this causes the results to drift from the true solution.

A simple method to reduce the step-size dependency could be to directly update Young's modulus in every iteration; however, this was found to increase the non-linearity in the model and analyses would converge poorly. An alternative approach is proposed: this involves updating Young's modulus for a controlled number of iterations at the start of each increment. Within each iteration, Young's modulus is frozen; this retains stability in the local iterative procedure.

It is acknowledged that the stiffness calculated as a result of the update of Young's modulus may be approximate, as the solution would not be converged. Therefore, some dependency on the last converged solution can be used by introducing a weighting factor, such that Young's moduli are a function of the last converged stress $\sigma_{\mathrm{LC}}$ and the stress from the previous iteration $\sigma_{\mathrm{LI}}-$ that is

\section{1. $E_{\mathrm{ur}}\left((1-r) \sigma_{\mathrm{LC}}+r \sigma_{\mathrm{LI}}\right)$}

where $r$ is the weighting factor which controls the dependency on the last converged stress. A value of $r=0$ forces the model to use only the last converged stress $\sigma_{\mathrm{LC}}$, and a value of $r=1$ forces the model to use only the stress from the last increment $\sigma_{\mathrm{LI}}$. This update is performed until the absolute relative change in Young's moduli $\Delta E$ between iterations reduces to below a given tolerance

72. $\Delta E=\frac{\left|E_{\mathrm{ur}}-\left(E_{\mathrm{ur}}\right)_{\mathrm{LI}}\right|}{E_{\mathrm{ur}}}$

As each of Young's moduli $E_{\mathrm{ur}}$ and $E_{\mathrm{i}}$ are linearly proportional to one another, any one of them may be used to determine the change in the moduli; in the proposed model, the unloaded modulus $E_{\mathrm{ur}}$ is selected.

Within a given increment, the tangent matrix can be considered to be consistent, in that it describes the infinitesimal relationship between stress and strain as defined in the rest of the model. However, from a more global perspective, it is more correct to describe this as a pseudo-consistent tangent because some of the variables are effectively frozen between iterations. 


\section{Results and discussions}

The HS-LC model has been implemented in finite-element analysis software Lusas for the purpose of solving boundary-value problems. Case studies with experimental data and predictions with other models were chosen to compare with the HS-LC model. The first problem is a one-dimensional oedometer test on a dry sand; experimental data, and predictions from the original HS model were extracted from the paper by Schanz et al. (1999). The second problem is a triaxial compression test on the same sand, again, experimental data and predictions from the original HS model are also available (Schanz et al., 1999). These two tests are chosen to demonstrate the capabilities of the HS-LC model in reproducing common soil laboratory tests. The parameters used in the HS-LC model for each analysis are shown in Table 1. The calibration parameters which these are adjusted to are: $E_{50}^{\text {ref }}=23890 \mathrm{kPa}, E_{\text {oed }}^{\text {ref }}=16500 \mathrm{kPa}, K_{0}=0.44$.

The results from previous experiments and predictions with the HS model (Schanz et al., 1999) were extracted through interpretation of graphs. Hence, it is expected that there may be some minor differences with these results. For the purposes of this study where two models are being compared at a broad level, these differences should be sufficiently insignificant.

Finally, a third set of analyses are performed on a hypothetical embankment using both a plane strain, and a 3D analysis. The factor of safety (FOS) of the slope is determined using $\varphi-c$ reduction. This result is compared against the FOS determined through analytical and graphical methods.

\subsection{Oedometer test}

This test considers the stress-strain response of confined uniaxial compression of loose Hostun sand (Table 1). The problem was modelled using a $4 \times 4$ grid of quadratic, quadrilateral axisymmetric elements. The oedometer sample

Table 1. Material parameters used in the HS-LC model simulations

\begin{tabular}{|c|c|c|}
\hline & Loose Hostun sand & Embankment soil \\
\hline$\rho: \mathrm{kg} / \mathrm{m}^{3}$ & & 1.4 \\
\hline$v$ & $0 \cdot 2$ & 0.35 \\
\hline$\varphi:^{\circ}$ & 34 & 30 \\
\hline$\psi:^{\circ}$ & $1 \cdot 5$ & 0.0 \\
\hline $\mathrm{c}: \mathrm{kPa}$ & 0.0 & $50 \cdot 0$ \\
\hline$m$ & 0.65 & 0.50 \\
\hline$R_{f}$ & 0.95 & 0.90 \\
\hline$E_{\text {ur }}^{\text {ref }} \mathrm{kPa}$ & 60000 & 60000 \\
\hline$E_{i}^{\text {reff }}: \mathrm{kPa}$ & 68913 & 25000 \\
\hline$K_{\mathrm{s}} / K_{\mathrm{c}}$ & 1.75 & $2 \cdot 00$ \\
\hline$\alpha$ & 0.959 & 1.000 \\
\hline$\sigma_{\mathrm{t}}: \mathrm{kPa}$ & 0 & 283 \\
\hline
\end{tabular}

modelled was of height and radius $3.5 \mathrm{~cm}$; the base of the soil was fully fixed and the outside vertical boundary was fixed in the horizontal direction. A prescribed displacement was applied to the top of the soil in the vertical direction, four unloading-reloading cycles were used and the analysis was performed using a total of 243 increments with a loading and unloading rate of $0.2 \mathrm{~mm}$ per increment. The full load curve for this test can be found in Figure 6 .

Experimental and predicted results are presented by Schanz et al. (1999). While a number of material parameters for the loose Hostun sand were described, and some material parameters were not available; including the oedometer modulus $E_{\text {oed. }}^{\text {ref }}$ For this study, the oedometer modulus was determined from the published experimental results by calculating the gradient of the oedometer curve at the reference stress $\sigma^{\text {ref }}=-100 \mathrm{kPa}$. The remaining stiffness parameters $E_{50}^{\text {ref }}$ and $E_{\mathrm{ur}}^{\mathrm{ref}}$ were determined by measuring the secant stiffness and unload-reload stiffness from the published triaxial data. The auxiliary parameters $\alpha$ and $K_{\mathrm{s}} / K_{\mathrm{c}}$ were calibrated to $E_{\text {oed }}$ and to an assumed value of the lateral earth pressure coefficient $K_{0}=1-\sin \varphi$. A small dilatancy angle was also used to reflect the volumetric strain increase observed in the experimental triaxial data. The full list of material parameters can be found in Table 1. The results of the oedometer simulation are plotted in Figure 7.

The prediction of the primary loading curve closely matches the experimental data below 0.02 axial strain. The published experimental reloading cycle is incomplete after this stage.

The unloading cycles initially under-predict the stiffness, however, the stiffness is predicted well in the third and fourth cycles. The experimental data show different paths between unloading and reloading; this is something not captured in the HS and HS-LC models because elastic stiffness is assumed when below the yield surface.

Another interesting feature of the HS and HS-LC predictions is the change in gradient towards the end of each unloading stage. This was found to be caused by the minor principal stress swapping orientation. This stress component is used to

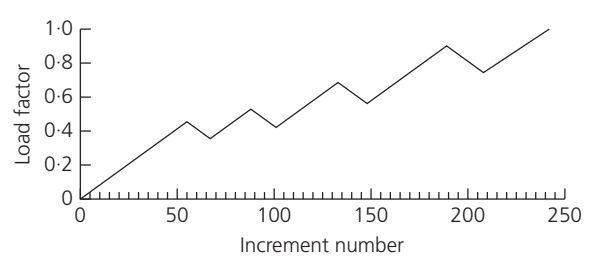

Figure 6. Load factor progression for the oedometer test 


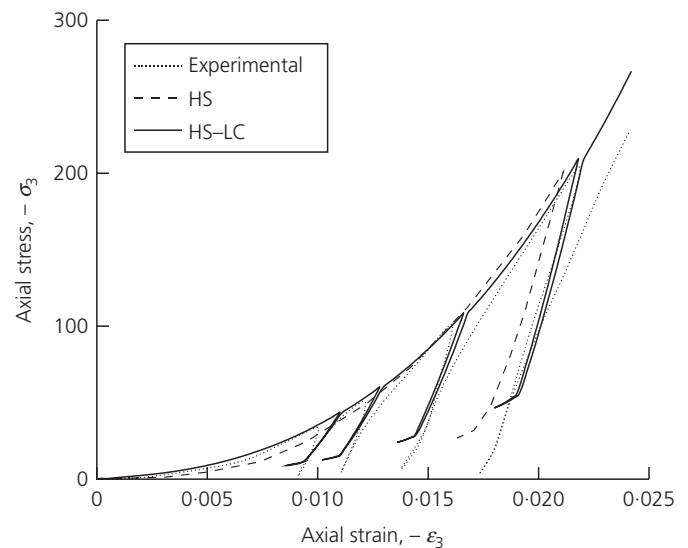

Figure 7. Oedometer test results and predictions for the HS model (source: Schanz et al., 1999), and the HS-LC model

calculate Young's modulus (Equation 11). It may be possible to remove this phenomenon in the HS and HS-LC models by using a stiffness dependency based on the mean stress.

As might be expected for a simple test, relatively few iterations are required to achieve convergence in each step, with each increment requiring only two iterations to converge to the given tolerances. The global tolerances used for this test, and all other tests in this study are as follows: the residual force norm tolerance is $0.001 \%$, and the incremental displacement norm tolerance is $0.001 \%$.

A second series of oedometer simulations were carried out to investigate the impact of step size in the HS-LC model. These analyses were stress controlled and the soil is taken to a vertical load of $100 \mathrm{kPa}$ using different step sizes.

Figure 8 shows that the model is step-size convergent when the load is split into at least ten steps. Minor differences in the response relate to the stiffness update procedure but the differences are considered negligible for the ten, 50 and 100 step tests. However, this procedure comes at a cost of increasing the total number of iterations per increment; this has been documented previously (Bower, 2017). As the stiffness of the problem changes, the global rate of convergence is lowered slightly. This effect is most prominent at the start of the test and can be seen in Figures 9-11, where the first few increments converge in four or more iterations. The remainder of each test converged in four increments or fewer, with most converging in two iterations. For the five step test, each of the increments took four iterations to converge.

Bower (2017) showed that use of the stiffness update procedure was necessary to preserve accuracy in the load paths of

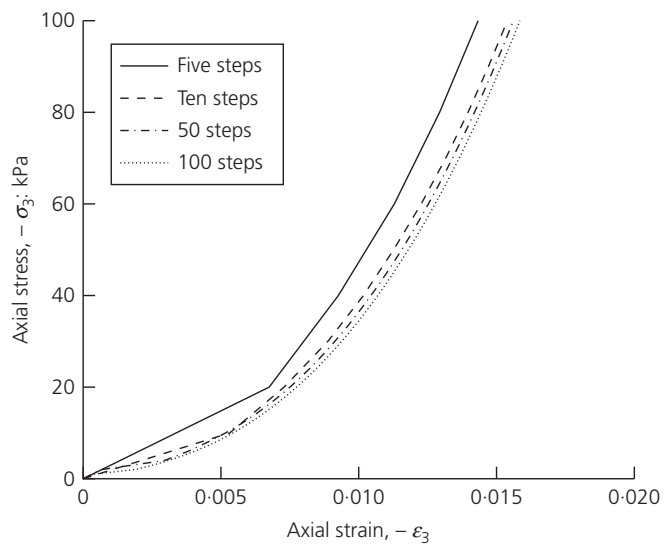

Figure 8. Oedometer test step-size study using stiffness update procedure with $r=0.33$; the stiffness was updated until $\Delta E \leq 0.05$

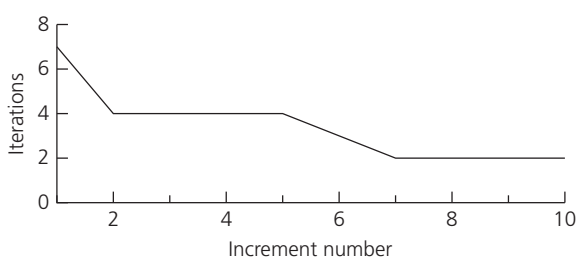

Figure 9. Number of iterations until convergence for the 10-step oedometer simulation

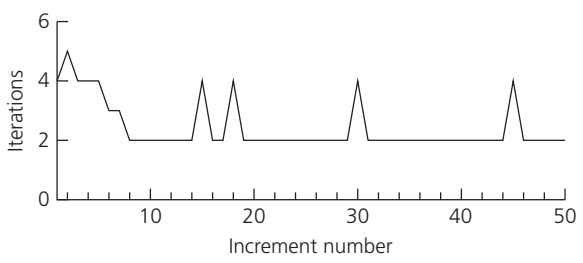

Figure 10. Number of iterations until convergence for the 50-step oedometer simulation

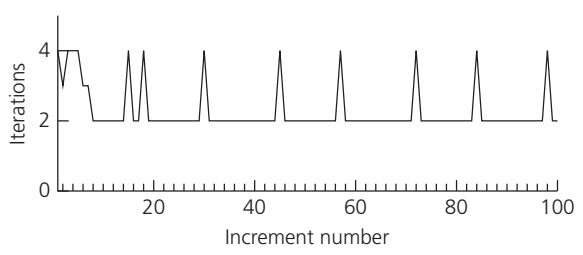

Figure 11. Number of iterations until convergence for the 100step oedometer simulation 
problems in which stress-dependent stiffness is an important factor. A similar comparison was performed with oedometer tests both with and without the stiffness update procedure. Without the update procedure, the oedometer tests using 250 and 500 steps produced similar load paths, however, the 50 and 100 step tests deviated from these other tests to a much greater degree than that shown in Figure 8.

\subsection{Triaxial compression test}

A drained, normally consolidated triaxial test was simulated using the same material properties as the oedometer test (Table 1). Experimental data and predictions from the original HS model were also found for this test (Schanz et al., 1999). The experimental and predicted shear response is plotted in Figure 12, and the volumetric responses are plotted in Figure 13.

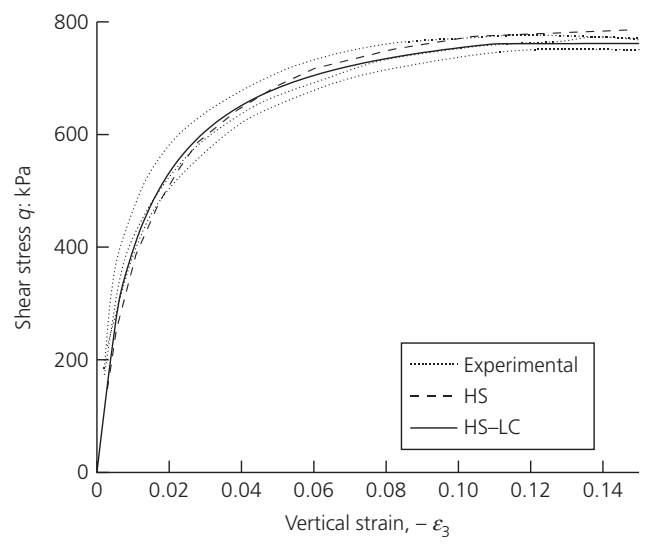

Figure 12. Triaxial test shear plots for simulation and three repeated experimental plots at $300 \mathrm{kPa}$ confining stress

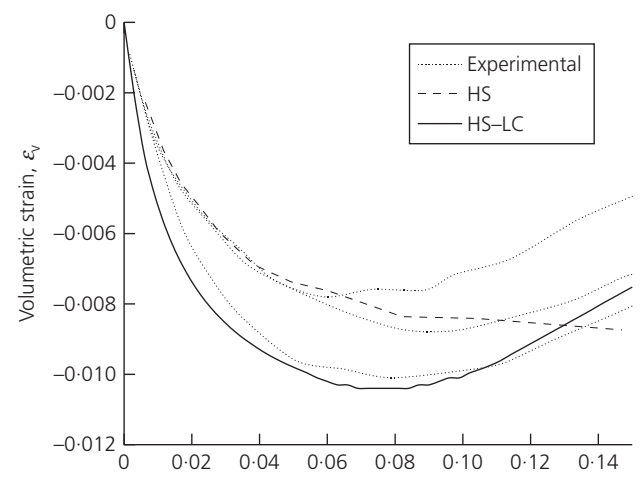

Figure 13. Triaxial test volumetric plots for simulation and three repeated experimental plots at $300 \mathrm{kPa}$ confining stress
The problem was analysed using a single axisymmetric element, the radius of the triaxial cell was taken to be $3 \mathrm{~cm}$ and the height to be $10 \mathrm{~cm}$. The base of the cell was fixed in the vertical direction and the axis of symmetry was fixed in the horizontal direction. The soil was prescribed an initial isotropic compressive stress of $300 \mathrm{kPa}$ to simulate the effective confining stress. A vertical displacement was applied to the top of the soil at a rate of $0.03 \mathrm{~cm}$ per increment for 50 increments.

The prediction of the shear stress response, as shown in Figure 12, follows a similar path to the original HS model, which slightly under-predicts the initial stiffness and the HS-LC provides a closer match. The shear stress failure limit for the HS-LC model can be seen to come into effect after $\varepsilon_{3}=-0.11$, and the maximum shear stress matches the original HS model and the experimental curves.

The predictions of the volumetric strain (Figure 13) vary between the HS and HS-LC models. The HS-LC model predicts an increased compaction at the start of the simulation compared to the experimental results and the HS model. Including the small dilatancy angle has caused the soil to dilate with increasing shear stress. The post-failure gradient matches the experimental results well. The modified Rowe dilatancy formula (Equation 13) is used in this simulation. The difference with the initial gradient between the two models is likely due to the dilatancy rules used in each model.

From the convergence plot for the triaxial test simulation (Figure 14), the first two increments converged in three iterations, this decreased to two iterations per increment until increment 20, where most increments converged in one iteration. On initial investigation with the original HS model, it was found that this test required an increasing number of iterations to converge as the test progressed, and a sharp increase in required iterations was observed as the shear stress approached the Mohr-Coulomb failure limit. By making the changes to the HS model proposed in this paper, this problem was overcome.

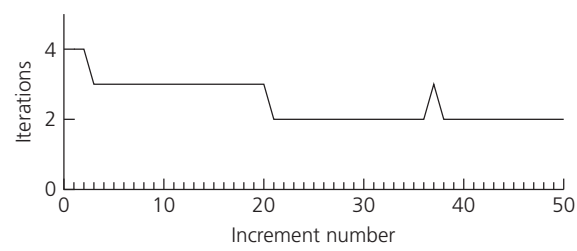

Figure 14. Number of iterations until convergence for the triaxial test 


\subsection{Slope stability analysis with $\varphi-c$ reduction}

In this section, the FOS of a slope due to its self-weight is determined using the $\varphi-c$ reduction technique, which is compared against an analytical and a graphical method.

The main principal of the $\varphi-c$ strength reduction method is to reduce the values of the friction angle $\varphi$ and cohesion $c$ such that the failure criterion is reduced in overall size. The parameters are reduced by the following relationships according to the FOS $F$

73. $c^{*}=\frac{c}{F}$

74. $\varphi^{*}=\tan \left(\frac{\arctan \varphi}{F}\right)$

where $c^{*}$ and $\varphi^{*}$ are the reduced set of failure parameters. In the HS-LC model, when the $\varphi-c$ code is activated, the hardening shear surface is deactivated and replaced with the Matsuoka-Nakai failure criterion in its final position. This is performed by setting $r_{\mathrm{q}}=R_{\mathrm{f}}$ in Equation 27. The stressdependent parameters $E_{\mathrm{i}}, E_{\mathrm{ur}}$ and $\sin \psi_{\mathrm{m}}$ are also frozen. Except for the dilatancy angle $\psi_{\mathrm{m}}$, which is set to the minimum of the frozen value of $\psi_{\mathrm{m}}$ and $\varphi^{*}$. The cap yield surface is also removed from the analysis during $\varphi-c$ reduction, as it merely describes the evolution of plastic variables rather than soil failure.

In Lusas, the FOS $F$ is adjusted using an automatic procedure until the stiffness of any part of the soil reaches a sufficiently small value (i.e. global failure).

In the following example, the height of the slope is $10 \mathrm{~m}$ and steepness is $45^{\circ}$. The mesh is extended $20 \mathrm{~m}$ beyond the end of the slope, which is considered sufficient to minimise boundary effects. The two-dimensional (2D) mesh (Figure 15) comprises

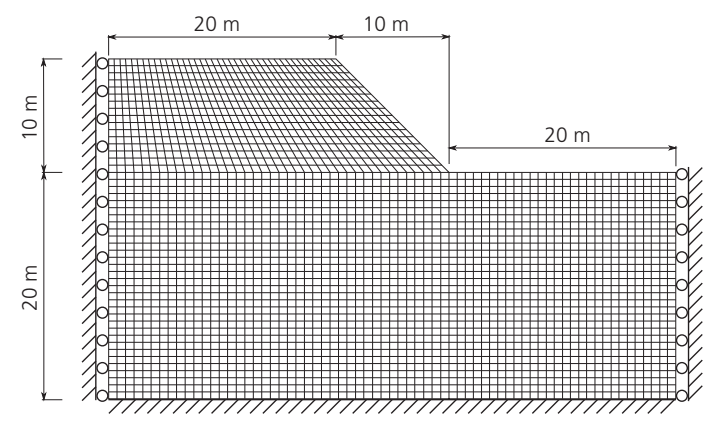

Figure 15. Mesh, geometry and boundary conditions of the plane-strain slope stability problem of 2933 nodes and 2816 linear, quadrilateral plane-strain elements.

The full HS-LC model was utilised in the initiation of stresses due to self-weight up until the point where the strength reduction procedure commenced, when the shear surface becomes locked to match the position of the failure surface.

The embankment was initialised in four equal lifts of $2.5 \mathrm{~m}$ each. First, the flat domain of the soil was initialised under gravity loading $\left(\boldsymbol{g}=9.807 \mathrm{~m} / \mathrm{s}^{2}\right)$. Each lift was then activated and gravity was applied to each layer as it was activated using automatic load incrementation. The Young modulus update described in Section 7 was not used here as it was determined to not have any influence on the final calculated FOS.

An analytical analysis of the same slope was performed using the ordinary method of slices (OMS). This analysis was performed using the WISE Uranium project slope stability calculator World Information Service on Energy (WISE, 2014), whereby the FOS at a series of centre points and radii were determined. The area around the minimum FOS was then scanned in more detail to further minimise the calculated FOS.

A graphical method for determining the FOS for a uniform, drained slope was also used (Steward et al., 2010). This is a non-iterative graphical method which produces two versions of the FOS; the first places emphasis on the cohesion $c$, and the second on the friction angle $\varphi$. The results from the analytical method, graphical method and $\varphi-c$ reduction method are shown in Table 2.

The calculated values of the FOS from each of the methods tested in this paper are broadly similar. From a geotechnical perspective, these values are close enough to be considered equivalent for design purposes. The FOS calculated through the $\varphi-c$ reduction method is slightly below those calculated through the other tested methods, making it a more conservative prediction.

From Figure 16, the failure mechanism of this slope is near circular, with the slip circle originating at the toe of the slope. From the OMS, the slip circle with the lowest FOS was determined to have a centre $15.0 \mathrm{~m}$ directly above the toe of the slope with a radius of $15.0 \mathrm{~m}$. The lower section of the HS-LC slip surface matches this well, however, the top section

Table 2. FOS for the slope calculated through different methods

\begin{tabular}{|cccc|}
\hline OMS & Graphical $(c)$ & Graphical $(\varphi)$ & $\varphi-c$ reduction \\
\hline 3.284 & 3.403 & 3.174 & 3.054 \\
\hline
\end{tabular}


A reformulated hardening soil model

Bower, Jefferson and Cleall

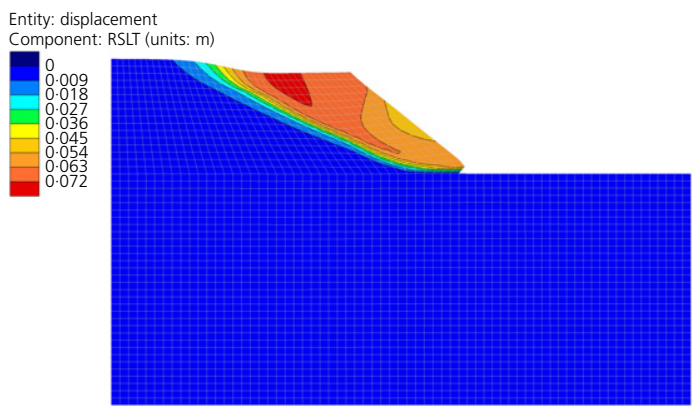

Figure 16. Resultant displacements for 2D plane-strain analysis at the end of $\varphi-c$ reduction, plotted at $20 \times$ exaggerated deformation

in the HS-LC analysis forms more of a wedge-type failure The OMS method was limited to circular slip surfaces; therefore, a closer match to the HS-LC results may be attainable with different slip surface shapes.

The contour plots of the shear strain $\gamma$ (Figure 17) show more precisely the location of shear failure in the soil. The highest shear strain can be found at a point about $5.0 \mathrm{~m}$ horizontal to a point just above the toe of the slope.

A second analysis of the slope was performed with modified boundary conditions. This particular case study is not included as a validation or benchmark but as a demonstration of the HS-LC model's 3D capabilities.

The 3D mesh (Figure 18) was formed of 12208 nodes and 10560 linear, eight-noded hexahedral elements. The boundary conditions were modified from plane-strain conditions such that one side of the 'slice' of slope was considered as a rough boundary (following from Griffiths and Marquez, 2007). The geometry of the slope and the initialisation of the soil

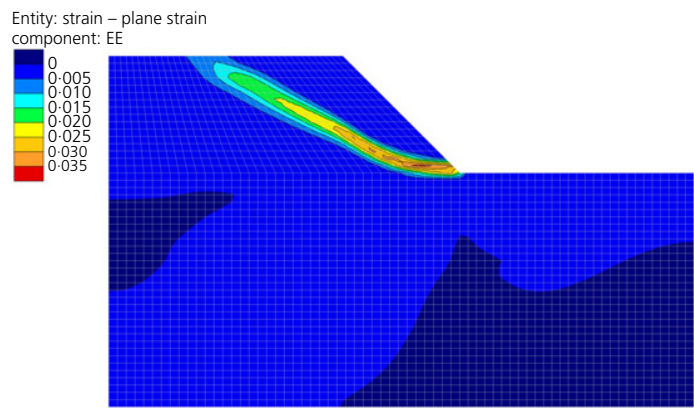

Figure 17. Shear strain for 2D plane-strain analysis at the end of $\varphi-c$ reduction

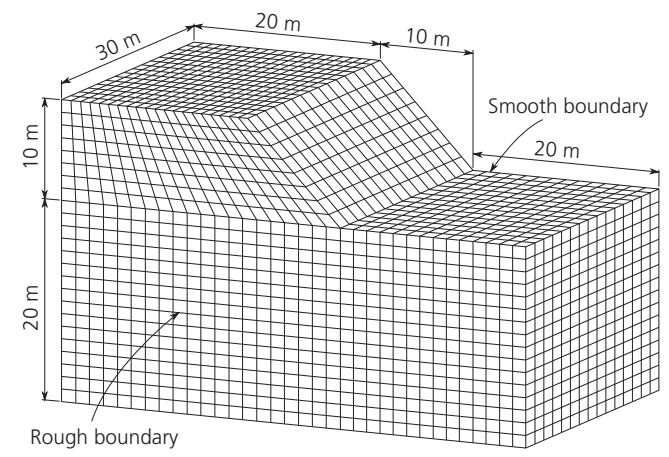

Figure 18. Mesh and geometry of the 3D slope stability problem. The base of the mesh is fully restrained, and the vertical sides of the mesh are restrained from deforming out of their respective planes; one of the vertical faces containing the slope is fixed in all directions

slope in layers was the same as that used in the 2D planestrain analysis, with a slice thickness of $30 \mathrm{~m}$. The smooth boundary on one side was used to enforce symmetry in the problem; thus, the slope analysed was $60 \mathrm{~m}$ wide, with rough boundaries on both ends.

The stresses were initialised in the soil with smooth boundaries on both sides. After the stresses were initialised, the displacements were reset and the boundary conditions modified such that one side was fully fixed. The strength reduction analysis was then initiated.

The inclusion of the rough boundary has modified the nature of the failure in the slope. From the displacement plot (Figure 19), the shape of the failure surface is ellipsoidal. With

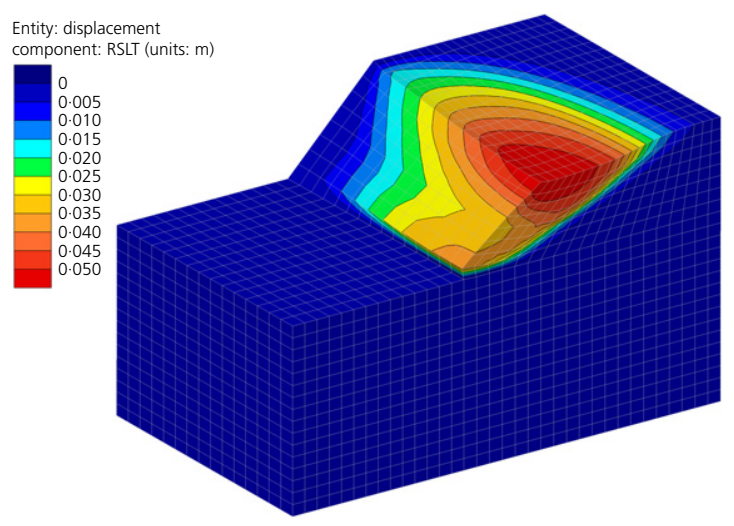

Figure 19. Resultant displacements for 3D rough-smooth boundary analysis at the end of $\varphi-c$ reduction, plotted at $40 \times$ exaggerated deformation 
the shear strain plot (Figure 20), the slip surface emerges at the toe of the slope near the smooth boundary.

The FOS calculated from the rough-smooth 3D analysis was 4.107. This is higher than the $2 \mathrm{D}$ plane-strain analysis, which assumes smooth-smooth boundaries. This indicates that the rough boundary assumption increases the calculated FOS for slope analyses.

The automatic incrementation of the $\varphi-c$ method in Lusas means that many trial safety factors are used. Only those which converged are included in the output. The solution continues until the change in FOS is sufficiently small. In these analyses, this tolerance was set to $0 \cdot 001$.

For the gravity initialisation phase in the 2D slope, each increment required between three and six iterations to converge to the given tolerances (Figure 21). In the 3D analysis (Figure 22), the initialisation increments required two or three iterations. In both analyses, the four lifts were performed using an automated incrementation procedure, which increases and

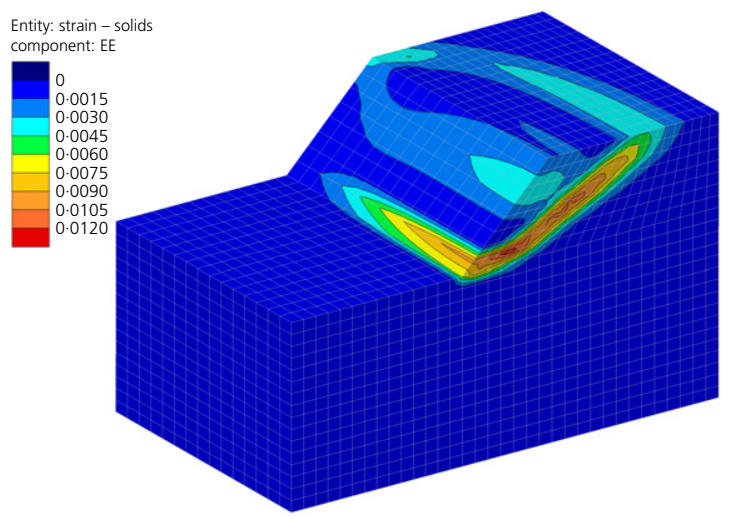

Figure 20. Shear strain for 3D rough-smooth boundary analysis at the end of $\varphi-c$ reduction

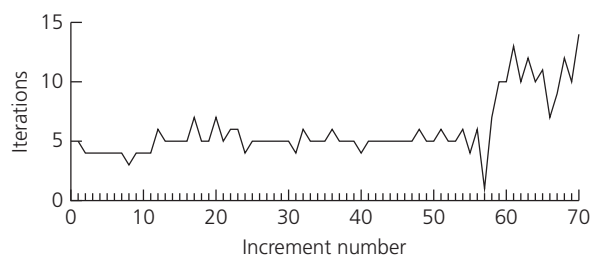

Figure 21. Number of iterations until convergence for 2D embankment analysis; increments 1-56 are the initialisation of gravity body forces in the slope, increment 57 is a displacement reset step, and the $\varphi-c$ reduction method begins at increment 58

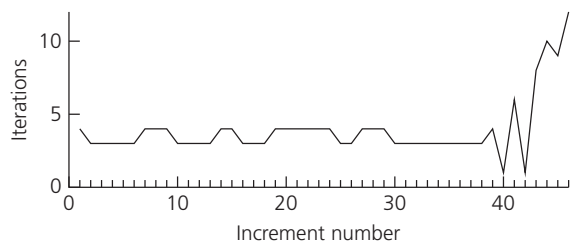

Figure 22. Number of iterations until convergence for $3 \mathrm{D}$ rough-smooth boundary embankment analysis; increments 1-39 are the initialisation of gravity body forces in the slope, increment 40 is a displacement reset step, the supports are modified at increment 41 , a second displacement reset step is used at increment 42 , and the $\varphi-c$ reduction method begins at increment 43

decreases depending on the number of global iterations required in the previous increment. Generally, each lift used between eight and ten incrementation steps for the $2 \mathrm{D}$ analysis, and between four and six incrementation steps for the 3D analysis. Between each lift, the calculated stresses were added to the equilibrium conditions and set as 'established' loads before the next lift was activated and gravity applied to it.

As the $\varphi-c$ method initialised for both analyses, the global solution generally took between five and 13 iterations to converge. This increase in required iterations is to be expected as certain elements in the soil are being made to fail, and the degree of non-linearity is increased.

To demonstrate the rate of convergence of the HS-LC model, one increment from each of the slope analyses was chosen and the residual force norm (also known as the norm of the out of balance forces) is plotted for each chosen iteration. For the 2D plane-strain analysis, increment 44 was selected (Figure 23) and for the 3D analysis, increment 46 was selected (Figure 24).

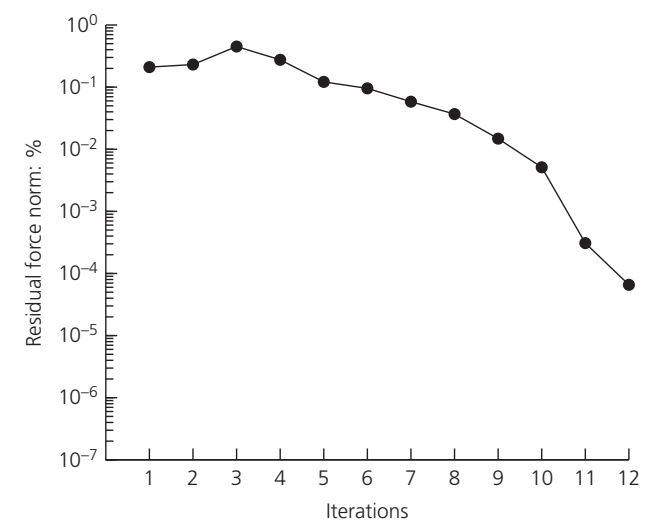

Figure 23. Residual force norm plot for increment 61 of the 2D plane-strain slope analysis 


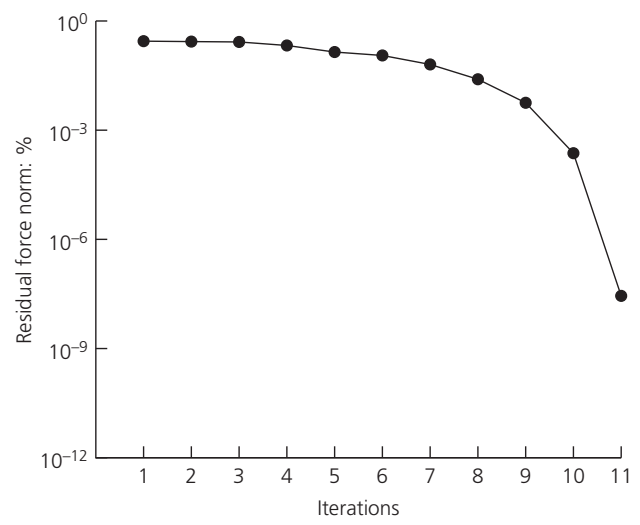

Figure 24. Residual force norm plot for increment 46 of the 3D rough-smooth slope analysis

The finite-element solution algorithm works to doubleprecision floating point accuracy.

It may be seen from Figure 23 that, after the first few iterations, convergence progresses quadratically, and reaches a value in the order of $10^{-7}$ very quickly. The case is similar for increment 46 of the 3D analysis (Figure 24) where the rate of convergence is initially in the order of $10^{-1}$ for a few iterations, and then accelerates dramatically to a value in the order of $10^{-11}$ by iteration 11 .

The rapid rates of convergence, and the acceleration towards convergence shows that the implementation of the consistent tangent matrix in the HS-LC model works as expected, and the quadratic rate of convergence of the Newton-Raphson method has been preserved.

Finally, a mesh convergence study was performed for the 2D plane-strain slope (Figure 25). The original mesh consisted of 2816 linear quadratic elements. A coarser mesh of 704 elements was generated by merging elements in groups of four, and a finer mesh of 11264 elements was generated by splitting each element into four equally sized smaller elements.

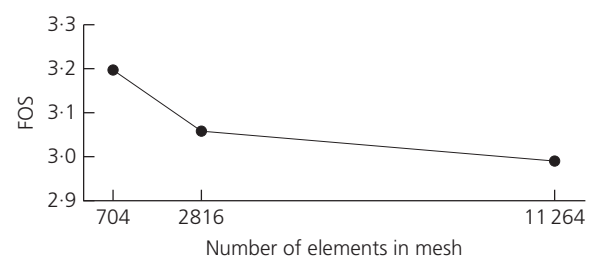

Figure 25. Mesh convergence plot of FOS for the 2D plane-strain analysis
The variable compared in the mesh convergence study was the final FOS determined by the $\varphi-c$ reduction method. The difference between the standard mesh and the coarse mesh is much greater than the difference between the standard mesh and the fine mesh. Therefore, it can reasonably be assumed that the mesh-converged solution for the FOS in this analysis is approximately $3 \cdot 0$.

The differences in the calculated FOS between mesh densities are likely to arise from the intersection of the slope and the base soil. This position is effectively a singularity, where a stress concentration can develop. The limitations of linear elements (i.e. shear locking) may also be contributing to some of the differences between meshes.

\section{Conclusions}

The implementation of the HS-LC model, with its new yield surface and hardening rule was able to reproduce results from the original HS model. The simulations using the new model were also a very good match for the laboratory-based experimental results, following experimental curves very closely.

Several limitations were found with the previous version of the HS model, the first was the formulation of the shear yield surface, which produces an asymptote when the magnitude of the trial shear stress is too high. This was addressed by reformulating the shear yield function such that it remains positive for all shear stress above yield.

The previous versions of the HS model used incremental relationships and did not take the residuals of the state variables into account during stress return mapping. The new formulation presented here uses total relationships, and does include these residuals, consequently, convergence rates for these simple laboratory simulations were found to be very good.

The use of a stiffness update procedure significantly reduced the amount of step-size dependency in the HS-LC model, allowing use of larger step sizes without causing the solution to drift. However, this was found to come at a cost of marginally increasing the required number of iterations per increment. The use of the stiffness update procedure can be easily optimised to reduce the computational cost of analyses.

Applications of the HS-LC to boundary-value problems were also tested with the analysis of a slope using $\varphi-c$ reduction in 2D. The convergence of this analysis was stable, and the calculated FOS closely matched that calculated through analytical and graphical methods. A second slope stability analysis was also performed in $3 \mathrm{D}$, with one of the boundaries considered 
as rough. This analysis predicted this FOS to be higher than the standard 2D plane-strain case.

Convergence rates for two particular iterations were also studied. The iterations chosen were at global soil failure and initially showed a slow and steady decrease in residual force norm, but the rate of convergence accelerated quickly as the iterations progressed; indicating that the quadratic rate of convergence of the Newton-Raphson method was preserved with the implementation of the consistent tangent matrix.

\section{Acknowledgements}

The authors acknowledge Lusas and Innovate UK for funding this work under KTP project 9082. In addition, special thanks are also made to Lusas for use of their software for this work.

\section{REFERENCES}

Benz T (2007) Small-strain Stiffness of Soils and its Numerical Consequences. PhD thesis, Universität Stuttgart, Institut für Geotechnik, Stuttgart, Germany.

Benz T, Wehnert M and Vermeer PA (2008) A Lode angle dependent formulation of the hardening soil model. In Proceedings of the 12th International Conference of International Association for Computer Methods and Advances in Geomechanics (Singh DN (ed.)). International Association for Computer Methods and Advances in Geomechanics (IACMAG), Goa, India, pp. 653-660.

Bower TA (2017) Constitutive Modelling of Soils and Fibre-Reinforced Soils. PhD thesis, Cardiff University, Cardiff, UK

Cocco LJ and Ruiz ME (2018) Numerical implementation of hardening soil model. In Numerical Methods in Geotechnical Engineering IX, Volume 1: Proceedings of the 9th European Conference on Numerical Methods in Geotechnical Engineering (NUMGE 2018) (Cardoso AS et al. (eds)), June 25-27, 2018, Porto, Portugal. CRC Press, London, UK, pp. 195-203.

Drucker DC, Prager W and Greenberg HT (1952) Extended limit design theorems for continuous media. Quarterly of Applied Mathematics 9(4): 381-389.

Duncan JM and Chang CY (1970) Nonlinear analysis of stress and strain in soils. Journal of the Soil Mechanics and Foundations Division 96(5): 1629-1653.

Griffiths D and Marquez R (2007) Three-dimensional slope stability analysis by elasto-plastic finite elements. Géotechnique 57(6): 537-546.

Jiang ZH and Zhang YX (2012) Second development of hardening soil constitutive model in FLAC ${ }^{3 \mathrm{~d}}$. Electronic Journal of Geotechnical Engineering 17(X): 3429-3439.

Lagioia R and Panteghini A (2014) The influence of the plastic potential on plane strain failure. International Journal for Numerical and Analytical Methods in Geomechanics 38(8): 844-862.

Li XS and Dafalias YF (2000) Dilatancy for cohesionless soils. Géotechnique 50(4): 449-460.

Marcher T and Vermeer PA (2001) Macromodelling of softening in non-cohesive soils. In Continuous and Discontinuous Modelling of Cohesive-Frictional Materials (Vermeer PA, Ehlers W, Herrmann H, Ramm E (eds)). Springer Science \& Business Media, Berlin, Germany, vol. 568, pp. 89-110.
Matsuoka H and Nakai T (1974) Stress-deformation and strength characteristics of soil under three different principal stresses. In Proceedings of the Japan Society of Civil Engineers. Japan Society of Civil Engineers, Tokyo, Japan, vol. 9, pp. 59-70

Obrzud RF (2010) On the use of the hardening soil small strain model in geotechnical practice. In Numerics in Geotechnics and Structures (Zimmermann Th, Truty A and Podleś K (eds)). Elmepress International, Lausanne, Switzerland, pp. 15-32.

Panteghini A and Lagioia R (2013) A fully convex reformulation of the original Matsuoka-Nakai failure criterion and its implicit numerically efficient integration algorithm. International Journal for Numerical and Analytical Methods in Geomechanics 38(6): 593-614.

Plaxis (2016) Material Models Manual. Plaxis, Delft, the Netherlands. Roscoe KH and Burland JB (1968) On the generalized stress-strain behaviour of wet clay. In Engineering Plasticity (Heyman J (ed.)). Cambridge University Press, Cambridge, UK, pp. 535-609.

Rowe PW (1962) The stress-dilatancy relation for static equilibrium of an assembly of particles in contact. Proceedings of the Royal Society of London A: Mathematical, Physical and Engineering Sciences 269(1339): 500-527.

Schanz T, Vermeer PA and Bonnier PG (1999) The hardening soil model: formulation and verification. In Beyond 2000 in Computational Geotechnics (Ronald BKB (ed.)). Balkema, Rotterdam, The Netherlands, pp. 281-296.

Simo JC and Hughes TJR (2006) Computational inelasticity. In Interdisciplinary Applied Mathematics (Marsden JE, Sirovich L and Wiggins S (eds)). Springer, New York, NY, USA, vol. 7.

Søreide OK (1990) Mixed Hardening Models for Frictional Soils. $\mathrm{PhD}$ thesis, Norwegian University of Science and Technology, Trondheim, Norway.

Steward T, Sivakugan N, Shukla S and Das B (2010) Taylor's slope stability charts revisited. International Journal of Geomechanics 11(4): 348-352.

Truty A and Obrzud R (2015) Improved formulation of the hardening soil model in the context of modeling the undrained behavior of cohesive soils. Studia Geotechnica et Mechanica 37(2): 61-68.

Wehnert M (2006) Ein Beitrag zur Drainerten und Undrainierten Analyse in der Geotechnik. PhD thesis, Universität Stuttgart, Institut für Geotechnik, Stuttgart, Germany.

WISE (World Information Service on Energy) (2014) Slope Stability Calculator. WISE, Amsterdam, the Netherlands. See http://www. wise-uranium.org/csst.html (accessed 15/10/2018).

Wood DM (1990) Soil Behaviour and Critical State Soil Mechanics. Cambridge University Press, Cambridge, UK.

\section{How can you contribute?}

To discuss this paper, please email up to 500 words to the editor at journals@ice.org.uk. Your contribution will be forwarded to the author(s) for a reply and, if considered appropriate by the editorial board, it will be published as discussion in a future issue of the journal.

Proceedings journals rely entirely on contributions from the civil engineering profession (and allied disciplines). Information about how to submit your paper online is available at www.icevirtuallibrary.com/page/authors, where you will also find detailed author guidelines. 